\title{
Kinetic derivation of diffuse-interface fluid models
}

\author{
Vincent Giovangigli $\odot^{*}$ \\ CMAP-CNRS, École Polytechnique, 91128 Palaiseau, France
}

(Received 20 April 2020; accepted 12 June 2020; published 6 July 2020)

\begin{abstract}
We present a full derivation of capillary fluid equations from the kinetic theory of dense gases. These equations involve van der Waals' gradient energy, Korteweg's tensor, and Dunn and Serrin's heat flux as well as viscous and heat dissipative fluxes. Starting from macroscopic equations obtained from the kinetic theory of dense gases, we use a second-order expansion of the pair distribution function in order to derive the diffuse interface model. The capillary extra terms and the capillarity coefficient are then associated with intermolecular forces and the pair interaction potential.
\end{abstract}

DOI: 10.1103/PhysRevE.102.012110

\section{INTRODUCTION}

Diffuse interface methods describe liquid-gas interfaces as transition zones with smooth variations of physical properties. Second gradient diffuse interface fluid models, also termed capillary fluids, have been successfully used to describe liquid-vapor interfaces, capillary waves, near-critical points, and three-phase contact lines, as well as droplets formation and nucleation [1-7]. The second gradient theory has been extended to binary fluids by Cahn and Hilliard allowing the modeling of spinodal decomposition [8]. Diffuse interface models may likewise be used in the supercritical domain allowing a smooth transition from supercritical to subcritical conditions [9]. These models are also compatible with the limiting free boundary problem when the interface thickness goes to zero [5].

The thermodynamics of diffuse interface models has been built by van der Waals [1] using a gradient squared term in the free energy, and the corresponding capillary tensor has been derived by Korteweg [2]. The proper heat flux has been obtained by Dunn and Serrin in the framework of rational thermodynamics [10]. These equations have alternatively been obtained from Hamiltonian considerations by Gavrilyuk and Shugrin [11], and their mathematical properties are discussed by Gavrilyuk and Gouin [12], Benzoni et al. [13], and Bresch et al. [14].

Aside from mechanical, thermodynamical, or mathematical arguments, it is also important to investigate capillary fluids at the molecular level. Statistical mechanics of equilibrium systems that are highly inhomogeneous on the scale of length of intermolecular forces has notably been used to investigate liquid-gas interfaces by Ono and Kondo [15], Evans [16], and Rowlinson and Widom [3]. The links between capillary phenomena and intermolecular forces have been deepened, and this has led to expressions for the interfacial energy, the capillary pressure tensor, and the capillary coefficient $[3,15,16]$.

\footnotetext{
*vincent.giovangigli@polytechnique.fr
}

Focusing on nonequilibrium models, the kinetic theory of gases has been used in various contexts in order to investigate phase transition as presented in the review paper by Frezzotti and Barbante [17]. Phase changes have notably been investigated by employing linearized Boltzmann equations with condensation-evaporation boundary conditions [18-20]. Boltzmann-Vlasov- and Enskog-Vlasov-type equations have been used to investigate more deeply spatial aspects of phase transition [21-32], as well as particulate flows [33]. Detailed molecular dynamics of Lennard-Jones fluids have further been performed by Frezzotti and Barbante and compared to capillary fluid models with a general very good agreement [7].

Focusing on the derivation of capillary fluids equations from the kinetic theory, a notable achievement was that of Rocard, who derived the capillary force acting locally in a fluid using a Taylor expansion of the two-point distribution function $[34,35]$. Rocard then recovered the Korteweg tensor and the capillary coefficient. Piechór has also investigated the links between kinetic nonlocal stresses and Korteweg's tensor using an Enskog-Vlasov equation [25]. A minimum BGK-Vlasov model has also been introduced by Takata and Noguchi [27] with a nonideal effect mediated through a nonlocal self-consistent force term and collisions acting as a thermal bath. A gradient-type energy has been obtained in the continuum limit as well as a Cahn-Hillard-type equation in an isothermal framework. To the best of the author's knowledge, however, a full derivation of nonisothermal capillary fluid equations from the kinetic theory of dense gases is still missing and is the object of this work.

The kinetic theory of dense gases is based on a generalized Boltzmann equation for the one-particle distribution function [36-42]. Such a Boltzmann-type equation is derived from the two first equations of the BBGKY hierarchy using cluster expansions and Bogoliubov's functional property. A key point in the generalized Boltzmann equation, allowing the derivation of capillary fluids, is that the distribution functions are evaluated at different spatial positions. In particular, models starting from the classical Boltzmann equation may lead to Burnett-type contributions but not to capillary fluids. The macroscopic equations are then obtained in the kinetic framework through an asymptotic expansion in terms of a uni- 
formity parameter analogous to that of the Chapman-Enskog method. The resulting macroscopic equations are found to be of the Navier-Stokes-Fourier type with density-dependent transport coefficients [36-42].

An examination of the asymptotic expansions then indicates that second-order corrections arising from the pair distribution function should be taken into account. A second-order expansion of the pair distribution function is then introduced and leads to the capillary fluid equations. The capillary energy, the Korteweg tensor, and the Dunn and Serrin heat flux are exactly recovered after lengthy calculations as well as an expression of the capillary coefficient due to van der Waals and Rayleigh. The capillary terms as well as the capillarity coefficient are related with intermolecular forces and the pair interaction potential.

The equations governing capillary fluids are presented in Sec. II. The kinetic theory of dense gases and the associated new asymptotics are described in Sec. III. A full derivation of capillary fluid equations from the kinetic theory is then given in Sec. IV.

\section{DIFFUSE INTERFACE FLUIDS}

We summarize in this section the equations governing fluids with diffuse interfaces also termed capillary fluids. These equations involve van der Waals' energy [1], Korteweg's tensor [2], and Dunn and Serrin's heat flux [10]. Incidentally, there exists another type of diffuse interface models of more empirical nature, namely, multifluids, typically obtained through averaging processes, that lie outside the scope of this work. We further discuss the ambiguity of rational themodynamics - the thermodynamics of irreversible processes-for such nonlinear systems of equations governing capillarity fluids, with the apparition of a gradients' product term in the rate of entropy production leading to an artificial alternative for the expression of transport fluxes.

\section{A. Van der Waals free energy}

The free energy per unit volume $\mathcal{A}$ in a second gradient theory is in the form

$$
\mathcal{A}=\mathcal{A}^{0}+\frac{1}{2} \varkappa|\nabla \rho|^{2},
$$

where $\mathcal{A}^{0}$ denotes the bulk free energy per unit volume, $\rho$ the mass density, $\nabla$ the usual differential operator, and $\varkappa$ the capillary coefficient. The superscript " 0 " is used to denote standard bulk phase thermodynamic properties that do not involve gradients. The free energy $\mathcal{A}^{0}$ depends only on the densities $\rho$ and the absolute temperature $T$, whereas the gradient squared term $\frac{1}{2} \varkappa|\nabla \rho|^{2}$ in $\mathcal{A}$ represents the excess free energy of the interfacial region [1-3,8]. We will assume in the following that the capillarity coefficient $\varkappa$ is constant for the sake of simplicity.

Denoting by $a=\mathcal{A} / \rho$ the free energy per unit mass, $T$ the absolute temperature, $s$ the entropy per unit mass, $p$ the pressure, and $v=1 / \rho$ the volume per unit mass, we have the classical thermodynamic relation $d a^{0}=-s^{0} d T-p^{0} d v$. Differentiating (1), we then obtain

$$
d a=-s^{0} d T-\left(p^{0}-\frac{1}{2} \varkappa|\nabla \rho|^{2}\right) d \nu+\frac{\varkappa \nabla \rho \cdot d \nabla \rho}{\rho},
$$

where $d$ denotes the differentiation operator. This shows that the entropy $s$ and the pressure $p$ are given by

$$
s=s^{0}, \quad p=p^{0}-\frac{1}{2} \varkappa|\nabla \rho|^{2} .
$$

The equality of the entropy $s$ with the bulk entropy $s^{0}$ is in agreement with the van der Waals derivation [1-3,8]. Denoting by $e$ the energy per unit mass and using the thermodynamic relation $e=a+T s$ we also get

$$
e=e^{0}+\frac{1}{2} \frac{\varkappa}{\rho}|\nabla \rho|^{2},
$$

and we denote by $\mathcal{E}=\rho e$ the energy per unit volume and $\mathcal{E}^{\mathrm{C}}=\frac{1}{2} \varkappa|\nabla \rho|^{2}$ its capillary part. The generalized Gibbs relation is finally in the form

$$
T d s=d e-\frac{p}{\rho^{2}} d \rho-\frac{1}{\rho} \varkappa \nabla \rho \cdot d \nabla \rho .
$$

We will deduce from (5) an entropy balance equation and next discuss entropy production and the structure of the momentum and energy fluxes.

In order to illustrate the impact of the van der Waals capillary energy, consider an equilibrium planar liquid-gas interface. The structure of the interface may be obtained by extremalizing the integral of entropy while energy and mass are conserved $[1,3,6]$. Denoting by 1 and $g$ the indices of the liquid and its vapor at equilibrium, we have $T_{1}=T_{\mathrm{g}}$, $p_{1}=p_{\mathrm{g}}$, and $g_{1}=g_{\mathrm{g}}$, where $g$ is the Gibbs function $g=$ $a+p / \rho$. Denoting by $z$ the normal coordinate, the interface is found to be isothermal and such that $\frac{1}{2} \varkappa(d \rho / d z)^{2}=\mathcal{A}-$ $\mathcal{A}_{\mathrm{g}}-g_{\mathrm{g}}\left(\rho-\rho_{\mathrm{g}}\right)$, and the density $\rho(z)$ may then be obtained by numerical integration $[1,3,43]$. The function $\mathcal{A}-\mathcal{A}_{\mathrm{g}}-$ $g_{\mathrm{g}}\left(\rho-\rho_{\mathrm{g}}\right)$ is the difference between $\mathcal{A}$ and its bitangent line, since $\mathcal{A}_{1}=\mathcal{A}_{\mathrm{g}}-g_{\mathrm{g}}\left(\rho_{\mathrm{l}}-\rho_{\mathrm{g}}\right)$ from the equality of pressures and since $\partial_{\rho} \mathcal{A}=g$ at constant temperature. Approximating the excess function $\mathcal{A}-\mathcal{A}_{\mathrm{g}}-g_{\mathrm{g}}\left(\rho-\rho_{\mathrm{g}}\right)$ as $\bar{A}\left(\rho-\rho_{\mathrm{l}}\right)^{2}(\rho-$ $\left.\rho_{\mathrm{g}}\right)^{2}$, where $\bar{A}$ is a constant, the density is found in the form $\rho(z)=\frac{1}{2}\left(\rho_{\mathrm{l}}+\rho_{\mathrm{g}}\right)+\frac{1}{2}\left(\rho_{\mathrm{l}}-\rho_{\mathrm{g}}\right) \tanh (z / 2 \bar{z})$ with the characteristic length $\bar{z}=(\varkappa / 2 \bar{A})^{1 / 2} /\left(\rho_{\mathrm{l}}-\rho_{\mathrm{g}}\right)$. Detailed numerical simulations have also shown that the error function erf yields accurate fits to actual density profiles [44].

\section{B. Equations of capillary fluids}

Using the pressure $p$ and the energy per unit mass $e$ given by (3) and (4), the equations governing capillary fluids may be written $[5,6]$

$$
\begin{gathered}
\partial_{t} \rho+\nabla \cdot(\rho \boldsymbol{v})=0, \\
\partial_{t}(\rho \boldsymbol{v})+\nabla \cdot(\rho \boldsymbol{v} \otimes \boldsymbol{v})+\nabla \cdot \mathcal{P}=0, \\
\partial_{t}\left[\rho\left(e+\frac{1}{2}|\boldsymbol{v}|^{2}\right)\right]+\nabla \cdot\left[\rho \boldsymbol{v}\left(e+\frac{1}{2}|\boldsymbol{v}|^{2}\right)\right] \\
+\nabla \cdot(\mathcal{Q}+\mathcal{P} \cdot \boldsymbol{v})=0,
\end{gathered}
$$

where $\partial_{t}$ denotes the time derivative operator, $\boldsymbol{v}$ the fluid velocity, $\mathcal{P}$ the pressure tensor, and $\mathcal{Q}$ the total heat flux. The transport fluxes $\mathcal{P}$ and $\mathcal{Q}$ contain capillary and dissipative 
terms

$$
\begin{aligned}
& \mathcal{P}=p \boldsymbol{I}+\varkappa \nabla \rho \otimes \nabla \rho-\varkappa \rho \Delta \rho \boldsymbol{I}+\mathcal{P}^{\mathrm{NS}}, \\
& \mathcal{Q}=\varkappa \rho \nabla \cdot \boldsymbol{v} \nabla \rho+\mathcal{Q}^{\mathrm{NS}},
\end{aligned}
$$

where $\boldsymbol{I}$ denotes the three-dimensional unit tensor, $\mathcal{P}^{\mathrm{NS}}$ the viscous pressure tensor, $\Delta=\nabla \cdot \nabla$ the Laplacian operator, and $\mathcal{Q}^{\mathrm{NS}}$ the Fourier heat flux [5,6]. The dissipative fluxes $\mathcal{P}^{\mathrm{NS}}$ and $\mathcal{Q}^{\mathrm{NS}}$ are classically of the Navier-Stokes and Fourier type [40,41,45-47]

$$
\begin{aligned}
& \mathcal{P}^{\mathrm{NS}}=-\mathfrak{v} \nabla \cdot \boldsymbol{v} \boldsymbol{I}-\eta\left(\nabla \boldsymbol{v}+\nabla \boldsymbol{v}^{t}-\frac{2}{3} \nabla \cdot \boldsymbol{v} \boldsymbol{I}\right), \\
& \mathcal{Q}^{\mathrm{NS}}=-\lambda \nabla T,
\end{aligned}
$$

where $\mathfrak{v}$ denotes the volume viscosity, $\eta$ the shear viscosity, and $\lambda$ the thermal conductivity.

We may decompose the total pressure tensor $\mathcal{P}$ and heat flux $\mathcal{Q}$ in the form $\mathcal{P}=p^{0} \boldsymbol{I}+\mathcal{P}^{\mathrm{NS}}+\mathcal{P}^{\mathrm{C}}$ and $\mathcal{Q}=\mathcal{Q}^{\mathrm{NS}}+$ $\mathcal{Q}^{\mathrm{C}}$ so that the Korteweg's pressure tensor $\mathcal{P}^{\mathrm{C}}$ and the Dunn and Serrin's heat flux $\mathcal{Q}^{\mathrm{C}}$ solely arise from capillarity

$$
\begin{aligned}
& \mathcal{P}^{\mathrm{C}}=-\frac{1}{2} \varkappa|\nabla \rho|^{2} \boldsymbol{I}+\varkappa \nabla \rho \otimes \nabla \rho-\varkappa \rho \Delta \rho \boldsymbol{I}, \\
& \mathcal{Q}^{\mathrm{C}}=\varkappa \rho \nabla \cdot \boldsymbol{v} \nabla \rho .
\end{aligned}
$$

The capillary part of the pressure $p^{\mathrm{C}}=-\frac{1}{2} \varkappa|\nabla \rho|^{2}$ has been included in the Korteweg tensor (13). Both capillary fluxes (13) and (14) will have to be recovered from the kinetic theory as well as the capillary energy per unit volume $\mathcal{E}^{\mathrm{C}}$. Such capillary fluids models have been successfully used for the study of many phase change problems including complex liquid-gas interfaces with topological changes [4-7,9].

\section{Ambiguity of rational thermodynamics}

Using Gibbs relation (5) and the governing equations (6)(8), the rate of entropy production may be written after some algebra

$$
\begin{aligned}
\rho\left(\partial_{t} s+\boldsymbol{v} \cdot \nabla s\right)+\nabla \cdot\left(\frac{\mathcal{Q}}{T}-\frac{\varkappa \rho \nabla \cdot v \nabla \rho}{T}\right) \\
=-\frac{1}{T}(\mathcal{P}-p \boldsymbol{I}-\varkappa \nabla \rho \otimes \nabla \rho+\varkappa \rho \Delta \rho \boldsymbol{I}): \nabla \boldsymbol{v} \\
\quad-(\mathcal{Q}-\varkappa \rho \nabla \cdot v \nabla \rho) \cdot \frac{\nabla T}{T^{2}},
\end{aligned}
$$

where $\mathbf{v}: \mathbf{w}$ denotes the full contraction between any two tensors $\mathbf{v}$ and $\mathbf{w}$. Proceeding as in the thermodynamics of irreversible processes [5,6,48], also termed rational thermodynamics, and using the Curie principle, we recover from (15) that $\mathcal{P}-p^{0} \boldsymbol{I}-\mathcal{P}^{\mathrm{C}}$ is given by (11) and similarly that $\mathcal{Q}-\mathcal{Q}^{\mathrm{C}}$ is given by (12), and we have recovered the expressions (9) and (10) of both fluxes $\mathcal{P}$ and $\mathcal{Q}$. Note also the absence of cross-effects because of the Curie principle [48]. The complete equations governing capillary fluids have thus been derived from rational thermodynamics by using the expression (15) for the rate of entropy production. Such a derivation has been presented in particular by Dunn and Serrin [10], Anderson et al. [5], and Jamet [6].

However, the situation is more complex than it may appear since there is a term in (15) involving the product of two gradients,

$$
\frac{1}{T^{2}} \varkappa \rho \nabla \cdot v \nabla \rho \cdot \nabla T .
$$

Considering this term as a temperature gradient term and regrouping it in $-(\mathcal{Q}-\rho \boldsymbol{\nabla} \cdot \boldsymbol{v} \varkappa \boldsymbol{\nabla} \rho) \cdot \boldsymbol{\nabla} T / T^{2}$, as in (15), we have recovered Korteweg's tensor and Dunn and Serrin's heat flux. However, using $\boldsymbol{\nabla} \cdot \boldsymbol{v}=\boldsymbol{\nabla} \boldsymbol{v}: \boldsymbol{I}$, this gradients' product term (16) could also be considered as a velocity derivative term. With such an interpretation, one may rewrite the rate of entropy production as

$$
\begin{aligned}
\rho\left(\partial_{t} s\right. & +\boldsymbol{v} \cdot \nabla s)+\nabla \cdot\left(\frac{\mathcal{Q}}{T}-\frac{\varkappa \rho \nabla \cdot \boldsymbol{v} \nabla \rho}{T}\right) \\
= & -\mathcal{Q} \cdot \frac{\nabla T}{T^{2}}-\frac{1}{T}(\mathcal{P}-p \boldsymbol{I}-\varkappa \nabla \rho \otimes \nabla \rho+\varkappa \rho \Delta \rho \boldsymbol{I} \\
& -\varkappa \rho \nabla \rho \cdot \nabla \log T \boldsymbol{I}): \nabla \boldsymbol{v} .
\end{aligned}
$$

Note that the temperature gradient arising from $\nabla(1 / T)$ then plays two different roles, as a gradient in $\mathcal{Q} \cdot \nabla T$ and as part of a flux in $\varkappa \rho \nabla \rho \cdot \nabla \log T \boldsymbol{I}$. Writing again the proportionality of fluxes and gradients with (17) we obtain different fluxes in an alternative unphysical form

$$
\begin{gathered}
\mathcal{P}^{\prime}=p \boldsymbol{I}+\varkappa \nabla \rho \otimes \nabla \rho-\varkappa \rho \Delta \rho \boldsymbol{I} \\
+\varkappa \rho \nabla \rho \cdot \nabla \log T \boldsymbol{I}+\mathcal{P}^{\mathrm{NS}} \\
\mathcal{Q}^{\prime}=\mathcal{Q}^{\mathrm{NS}} .
\end{gathered}
$$

Therefore, depending on the expression of the rate of entropy production (15) or (17), one may either obtain the correct fluxes (9) and (10) or the alternative fluxes (18) and (19). These alternative fluxes (18) and (19) have notably been proposed by Freistühler and Kotschote [49], Heida and Málek who obtained both sets of fluxes [50], and Souček et al. [51], who have introduced a linear combination of both fluxes.

Rational thermodynamics, which is usually a faithful tool for linear-like problems, is here unable to determine unambiguously the proper transport fluxes. As quoted by de Groot and Mazur, the methods of rational thermodynamics are essentially valid for linear relations between fluxes and gradients [48]. For nonlinear systems like capillary fluids, there is a failure of rational thermodynamics in the sense that a simple algebraic reordering of the entropy production rate from (15) into (17) seems to lead to different physical fluxes (9) and (10) or (18) and (19) and thus to different physics. We thus conclude that only finer physical theories may determine unambiguously the proper fluxes and equations. The correct fluxes (9) and (10) have been obtained in particular by Gavrilyuk and Shugrin [11] from Hamilton's principle, they have important mathematical properties [12,13] and are obtained from the kinetic theory of dense gases in this work, thereby resolving the alternative. The alternative fluxes (18) and (19) finally appear to be unphysical as was overlooked in the literature [49-51] because of the ambiguity of rational mechanics.

\section{A DENSE GAS KINETIC MODEL}

A kinetic theory describing moderately dense gases is summarized in this section as well as the corresponding 
macroscopic dense fluid equations. The traditional first-order expansion in terms of a uniformity parameter is presented and a second-order expansion of the pair distribution function is introduced, as required by the capillary terms.

\section{A. Kinetic framework}

We consider a single dense gas and the associated BBGKY hierarchy governing multiple-particle distribution functions as presented in Chapman and Cowling [40], Ferziger and Kaper [41], and Lifshitz and Pitaevskii [52]. Assuming for the sake of simplicity that there are no external forces acting on the particles, the two first equations of the BBGKY hierarchy are in the form

$$
\begin{gathered}
\partial_{t} f_{1}+\boldsymbol{c}_{1} \cdot \nabla_{\mathbf{r}_{1}} f_{1}=\int \theta_{12} f_{2} d \boldsymbol{x}_{2}, \\
\partial_{t} f_{2}+\boldsymbol{c}_{1} \cdot \nabla_{\mathbf{r}_{1}} f_{2}+\boldsymbol{c}_{2} \cdot \nabla_{\mathbf{r}_{2}} f_{2}-\theta_{12} f_{2} \\
=\int\left(\theta_{13}+\theta_{23}\right) f_{3} d \boldsymbol{x}_{3},
\end{gathered}
$$

where $\partial_{t}$ denotes the time derivative operator, $\mathbf{r}_{i}$ the spatial coordinates of the $i$ th particle, $\boldsymbol{c}_{i}$ the velocity of the $i$ th particle, $f_{k}$ the $k$ particle(s) distribution function, $\nabla_{\mathbf{r}_{i}}$ the derivative operator with respect to the spatial coordinate $\mathbf{r}_{i}$, and $\nabla_{\boldsymbol{c}_{i}}$ the derivative operator with respect to the velocity $\boldsymbol{c}_{i}$. We denote for short by $\boldsymbol{x}_{i}$ the pair of vectors $\boldsymbol{x}_{i}=\left(\mathbf{r}_{i}, \boldsymbol{c}_{i}\right)$ and by $d \boldsymbol{x}_{i}$ the volume element $d \mathbf{r}_{i} d \boldsymbol{c}_{i}$. When there is no ambiguity, we will also denote by $\mathbf{r}$ and $\boldsymbol{c}$ the vectors $\mathbf{r}_{1}$ and $\boldsymbol{c}_{1}$ and by $\nabla$ the differential operator $\nabla_{\mathbf{r}_{1}}$. The differential operator $\theta_{i j}$ is given by

$$
\theta_{i j}=\frac{1}{m} \nabla_{\mathbf{r}_{i}} \varphi_{i j} \cdot \nabla_{\boldsymbol{c}_{i}}+\frac{1}{m} \nabla_{\mathbf{r}_{j}} \varphi_{i j} \cdot \nabla_{\boldsymbol{c}_{j}}
$$

where $m$ denotes the particle mass and $\varphi_{i j}$ the interaction potential between the $i$ th and $j$ th particles that depends only on the intermolecular distance $r_{i j}=\left|\mathbf{r}_{j}-\mathbf{r}_{i}\right|$. The traditional one-particle distribution function is then $f_{1}=f_{1}\left(\boldsymbol{x}_{1}, t\right)=$ $f_{1}\left(\mathbf{r}_{1}, \boldsymbol{c}_{1}, t\right)$, and the particle pair distribution function $f_{2}=$ $f_{2}\left(\boldsymbol{x}_{1}, \boldsymbol{x}_{2}, t\right)$ is also symmetric $f_{2}\left(\boldsymbol{x}_{1}, \boldsymbol{x}_{2}, t\right)=f_{2}\left(\boldsymbol{x}_{2}, \boldsymbol{x}_{1}, t\right)$. The triplet particle distribution function $f_{3}$ is assumed to be zero in this work for the sake of simplicity. These equations are the two first equations of the BBGKY hierarchy that has been derived independently by Bogoliubov [53], Born and Green [54], and Kirkwood [55] and introduced by Yvon [56], and we refer to the literature [40,41,52] for more details.

The aim of this work being the derivation of the macroscopic equations governing diffuse interface fluids, we use only a simplified version of the kinetic theory of moderately dense gases. In particular, triple collisions are not taken into account, although they may indeed be included in the model, and some of the difficulties associated with dense gases are not detailed like bound states, divergences with quadruple collisions in three dimensions, $\mathrm{H}$ theorems, or long-time tails for time correlation functions, and we refer to the literature [36-42,52-57].

\section{B. Macroscopic equations}

The gas number density $n_{1}$ is given by

$$
n_{1}\left(\mathbf{r}_{1}, t\right)=\int f_{1}\left(\mathbf{r}_{1}, \boldsymbol{c}_{1}, t\right) d \boldsymbol{c}_{1}
$$

and will also be denoted by $n$ when there is no ambiguity, and the pair number density $n_{2}$ reads

$$
n_{2}\left(\mathbf{r}_{1}, \mathbf{r}_{2}, t\right)=\int f_{2}\left(\mathbf{r}_{1}, \boldsymbol{c}_{1}, \mathbf{r}_{2}, \boldsymbol{c}_{2}, t\right) d \boldsymbol{c}_{1} d \boldsymbol{c}_{2} .
$$

The mass density is $\rho=m n$, and the gas velocity $\boldsymbol{v}$ reads

$$
\rho \boldsymbol{v}\left(\mathbf{r}_{1}, t\right)=\int m \boldsymbol{c}_{1} f_{1}\left(\mathbf{r}_{1}, \boldsymbol{c}_{1}, t\right) d \boldsymbol{c}_{1} .
$$

The internal energy per unit mass of the fluid is decomposed into

$$
e=e^{\mathrm{K}}+e^{\mathrm{P}}
$$

where $e^{\mathrm{K}}$ is the kinetic part that originates from the peculiar motion of the particles, whereas $e^{\mathrm{P}}$ is the potential part due to the pair interaction potential $[40,41]$. We will denote by $\mathcal{E}=\rho e$ and $\mathcal{E}^{\mathrm{P}}=\rho e^{\mathrm{P}}$ the corresponding energy densities per unit volume. The internal energy density $e^{\mathrm{K}}$ and the potential part $e^{\mathrm{P}}$ may be written

$$
\begin{aligned}
\rho e^{\mathrm{K}} & =\int \frac{1}{2} m\left|\boldsymbol{c}_{1}-\boldsymbol{v}\right|^{2} f_{1}\left(\mathbf{r}_{1}, \boldsymbol{c}_{1}, t\right) d \boldsymbol{c}_{1}, \\
\rho e^{\mathrm{P}} & =\int \frac{1}{2} \varphi_{12}\left(r_{12}\right) n_{2}\left(\mathbf{r}_{1}, \mathbf{r}_{2}, t\right) d \mathbf{r}_{2},
\end{aligned}
$$

where the interaction potential $\varphi_{12}$ depends only on $r_{12}=$ $\left|\mathbf{r}_{2}-\mathbf{r}_{1}\right|$.

The general macroscopic equations in unclosed form are further obtained by taking appropriate moments of (20) and (21). The mass conservation equation is obtained by integrating (20) with respect to $c_{1}$ and reads

$$
\partial_{t} \rho+\nabla \cdot(\rho \boldsymbol{v})=0
$$

The momentum equation, obtained by multiplying (20) by $m c_{1}$ and integrating with respect to $c_{1}$, is found in the form

$$
\partial_{t}(\rho \boldsymbol{v})+\nabla \cdot(\rho \boldsymbol{v} \otimes \boldsymbol{v})+\nabla \cdot\left(\mathcal{P}^{\mathrm{K}}+\mathcal{P}^{\mathrm{P}}\right)=0,
$$

where $\mathcal{P}^{\mathrm{K}}$ is the traditional kinetic part of the pressure tensor

$$
\mathcal{P}^{\mathrm{K}}\left(\mathbf{r}_{1}, t\right)=\int m\left(\boldsymbol{c}_{1}-\boldsymbol{v}\right) \otimes\left(\boldsymbol{c}_{1}-\boldsymbol{v}\right) f_{1}\left(\mathbf{r}_{1}, \boldsymbol{c}_{1}, t\right) d \boldsymbol{c}_{1},
$$

whereas $\mathcal{P}^{\mathrm{P}}$ is the potential part

$$
\begin{gathered}
\mathcal{P}^{\mathrm{P}}\left(\mathbf{r}_{1}, t\right)=-\frac{1}{2} \int \frac{\varphi_{12}^{\prime}\left(r_{12}\right)}{r_{12}} \mathbf{r}_{12} \otimes \mathbf{r}_{12} n_{2}\left[\mathbf{r}_{1}-(1-\alpha) \mathbf{r}_{12},\right. \\
\left.\mathbf{r}_{1}+\alpha \mathbf{r}_{12}, t\right] d \alpha d \mathbf{r}_{12},
\end{gathered}
$$

where the scalar $\alpha$ must be integrated over $(0,1)$. The tensor $\mathcal{P}^{\mathrm{K}}$ corresponds to the transfer of momentum due to the flow of particles and $\mathcal{P}^{\mathrm{P}}$ to the transfer of momentum between particles due to intermolecular forces $[40,41]$.

The energy conservation equation is next derived in several steps. One first multiplies (20) by $\frac{1}{2} m\left|\boldsymbol{c}_{1}-\boldsymbol{v}\right|^{2}$ and integrates with respect to $c_{1}$ in order to obtain a balance equation for the kinetic part $e^{\mathrm{K}}$ of the internal energy. The balance equation 
for the potential part of the energy $e^{\mathrm{p}}$ is then obtained by multiplying the two-point distribution function equation (21) by the potential energy $\frac{1}{2} \varphi_{12}\left(r_{12}\right)$ and integrating with respect to $c_{1}, \boldsymbol{c}_{2}$, and $\mathbf{r}_{12}=\mathbf{r}_{2}-\mathbf{r}_{1}$. A balance equation for the kinetic energy $\frac{1}{2}|\boldsymbol{v}|^{2}$ is also obtained by taking the scalar product of (30) by $\boldsymbol{v}$ and added to the previous equations. After lengthy calculations, the governing equation for $e+\frac{1}{2}|\boldsymbol{v}|^{2}=$ $e^{\mathrm{K}}+e^{\mathrm{P}}+\frac{1}{2}|\boldsymbol{v}|^{2}$ is found in the form

$$
\begin{aligned}
& \partial_{t}\left[\rho\left(e+\frac{1}{2}|\boldsymbol{v}|^{2}\right)\right]+\nabla \cdot\left[\rho\left(e+\frac{1}{2}|\boldsymbol{v}|^{2}\right)\right] \\
& \quad+\nabla \cdot\left[\mathcal{Q}^{\mathrm{K}}+\mathcal{Q}^{\mathrm{P}}+\left(\mathcal{P}^{\mathrm{K}}+\mathcal{P}^{\mathrm{P}}\right) \cdot \boldsymbol{v}\right]=0,
\end{aligned}
$$

where

$$
\begin{aligned}
& \mathcal{Q}^{\mathrm{K}}\left(\mathbf{r}_{1}, t\right)=\frac{1}{2} \int m\left|\boldsymbol{c}_{1}-\boldsymbol{v}\right|^{2}\left(\boldsymbol{c}_{1}-\boldsymbol{v}\right) f_{1}\left(\mathbf{r}_{1}, \boldsymbol{c}_{1}, t\right) d \boldsymbol{c}_{1} \\
& \text { and } \mathcal{Q}^{\mathrm{P}}=\mathcal{Q}_{1}^{\mathrm{P}}+ \mathcal{Q}_{2}^{\mathrm{P}} \text { with } \\
& \mathcal{Q}_{1}^{\mathrm{P}}\left(\mathbf{r}_{1}, t\right)= \frac{1}{2} \int \varphi_{12}\left(r_{12}\right)\left(\boldsymbol{c}_{1}-\boldsymbol{v}\right) f_{2}\left(\mathbf{r}_{1}, \boldsymbol{c}_{1}, \mathbf{r}_{2}, \boldsymbol{c}_{2}, t\right) \\
& \times d \boldsymbol{c}_{1} d \mathbf{r}_{2} d \boldsymbol{c}_{2}, \\
& \mathcal{Q}_{2}^{\mathrm{P}}\left(\mathbf{r}_{1}, t\right)=- \frac{1}{4} \int \frac{\varphi_{12}^{\prime}\left(r_{12}\right)}{r_{12}} \mathbf{r}_{12} \mathbf{r}_{12} \cdot\left(\boldsymbol{c}_{1}-\boldsymbol{v}+\boldsymbol{c}_{2}-\boldsymbol{v}\right) \\
& \times f_{2}\left[\mathbf{r}_{1}-(1-\alpha) \mathbf{r}_{12}, \boldsymbol{c}_{1}, \mathbf{r}_{1}+\alpha \mathbf{r}_{12}, \boldsymbol{c}_{2}, t\right] \\
& \times d \alpha d \boldsymbol{c}_{1} d \mathbf{r}_{12} d \boldsymbol{c}_{2},
\end{aligned}
$$

and the scalar $\alpha$ must be integrated over $(0,1)$. The heat flow vector $\mathcal{Q}^{\mathrm{K}}$ is similar to that of a dilute gas and represents the transfer of thermal energy due to the flow of molecules. The first potential part $\mathcal{Q}_{1}^{\mathrm{p}}$ represents the flow of potential energy carried by the molecules, and $\mathcal{Q}_{2}^{\mathrm{p}}$ represents the flux of energy associated with long-range forces and thermal agitation. More specifically, the scalar product of $\mathcal{Q}_{2}^{\mathrm{p}}$ with a normal vector to a surface element represents the work per unit time done on molecules on one side, which move with their peculiar motion, by the molecules on the other side, and both fluxes $\mathcal{Q}_{1}^{\text {p }}$ and $\mathcal{Q}_{2}^{\text {p }}$ depend only on the peculiar velocities $\boldsymbol{c}_{1}-\boldsymbol{v}$ and $\boldsymbol{c}_{2}-\boldsymbol{v}$.

\section{Generalized Boltzmann equation}

The kinetic theory of dense gases involves a generalized Boltzmann equation for the one-particle distribution function $f_{1}$ that may be derived by using cluster expansions and Bogoliubov's method [36-42]. To this aim, the two-point distribution function $f_{2}$ is written in the form

$$
f_{2}=\mathfrak{S}_{12} f_{1}\left(\boldsymbol{x}_{1}, t\right) f_{1}\left(\boldsymbol{x}_{2}, t\right) \text {, }
$$

where the combined streaming operator $\mathfrak{S}_{12}$ is given by

$$
\mathfrak{S}_{12}=\exp \left(-t \mathfrak{H}_{12}\right) \exp \left(t \mathfrak{H}_{1}\right) \exp \left(t \mathfrak{H}_{2}\right) .
$$

The operator $\mathfrak{H}_{i}$ denotes the Hamiltonian operator of the $i$ th particle alone, $\mathfrak{H}_{i}=\boldsymbol{c}_{i} \cdot \nabla_{\mathbf{r}_{i}}$, whereas

$$
\mathfrak{H}_{12}=\boldsymbol{c}_{1} \cdot \nabla_{\mathbf{r}_{1}}+\boldsymbol{c}_{2} \cdot \nabla_{\mathbf{r}_{2}}-\theta_{12}
$$

denotes the Hamiltonian operator for the particle pair. In the expression (37) the contribution arising from triple distribution products or more $[38,41]$ has been neglected for the sake of simplicity. The streaming operators $\exp \left(t \mathfrak{H}_{s}\right)$ form
Abelian one-parameter groups of operators and correspond to the streaming of systems of $s$ particles under the influence of the Hamiltonian $\mathfrak{H}_{s}$. It has also been assumed that the initial distribution is a product of uncorrelated distributions $f_{2}\left(\boldsymbol{x}_{1}, \boldsymbol{x}_{2}, 0\right)=f_{1}\left(\boldsymbol{x}_{1}, 0\right) f_{1}\left(\boldsymbol{x}_{2}, 0\right)$. A fundamental assumption in the model is that the operator $\mathfrak{S}_{12}\left(\boldsymbol{x}_{1}, \boldsymbol{x}_{2}, t\right)$ as a finite limit $\mathfrak{S}_{12}^{\infty}\left(\boldsymbol{x}_{1}, \boldsymbol{x}_{2}\right)$ as $t$ is large with respect to the collision time; we refer to Choh and Uhlenbeck [36], Andrews [37], García Colín et al.[38], Ferziger and Kaper [41], and Dorfman and van Beijeren [42] for more details.

The generalized Boltzmann equation governing the oneparticle distribution function $f_{1}$ is then in the form

$$
\partial_{t} f_{1}+\boldsymbol{c}_{1} \cdot \nabla_{\mathbf{r}_{1}} f_{1}=J\left(f_{1}, f_{1}\right)
$$

where

$$
J\left(f_{1}, f_{1}\right)=\int \theta_{12} \mathfrak{S}_{12}^{\infty} f_{1}\left(\boldsymbol{x}_{1}, t\right) f_{1}\left(\boldsymbol{x}_{2}, t\right) d \boldsymbol{x}_{2} .
$$

A key point in (39) is that the particles are taken at different positions $\mathbf{r}_{1}$ and $\mathbf{r}_{2}$, and Taylor expansion will be required in order to localize the collision operator.

\section{Chapman-Enskog expansion}

The asymptotic method that leads to the macroscopic fluid equations in the kinetic theory of dense gases is analogous to that of Chapman-Enskog. The distribution $f_{1}$ is expanded as a power series in terms of a uniformity parameter $\mu$, which is a measure of the gradients of the macroscopic variables. Such an expansion is valid in the hydrodynamic stage where $f_{1}$ depends only on time through the macroscopic variables $n$, $\boldsymbol{v}$, and $T$. The macroscopic variables, on the other hand, are governed by the hydrodynamic equations [36-42].

In the Navier-Stokes regime, the one-particle distribution is expanded in the form

$$
f_{1}=f_{1}^{(0)}+\mu f_{1}^{(1)}+O\left(\mu^{2}\right),
$$

in terms of the uniformity parameter $\mu$. The case where $\mu=0$ corresponds to a local uniform state, and this implies a firstorder expansion for $f_{1}\left(\mathbf{r}_{1}^{\prime}, \boldsymbol{c}_{1}\right)$ around $\left(\mathbf{r}_{1}, \boldsymbol{c}_{1}\right)$ with $\delta \mathbf{r}_{1}=\mathbf{r}_{1}^{\prime}-$ $\mathbf{r}_{1}$ in the form

$$
\begin{aligned}
f_{1}\left(\mathbf{r}_{1}^{\prime}, \boldsymbol{c}_{1}\right)= & f_{1}^{(0)}\left(\mathbf{r}_{1}, \boldsymbol{c}_{1}\right)+\mu\left[f_{1}^{(1)}\left(\mathbf{r}_{1}, \boldsymbol{c}_{1}\right)\right. \\
& \left.+\nabla_{\mathbf{r}_{1}} f_{1}^{(0)}\left(\mathbf{r}_{1}, \boldsymbol{c}_{1}\right) \cdot \delta \mathbf{r}_{1}\right]+O\left(\mu^{2}\right) .
\end{aligned}
$$

The collision operator $J$ acting on a distribution $h$ is similarly expanded locally as

$$
J(h, h)=J_{0}(h, h)+\mu J_{1}\left(h, \nabla_{\mathbf{r}_{1}} h\right)+O\left(\mu^{2}\right),
$$

where $J_{0}$ is the usual Boltzmann collision operator and $J_{1}$ is a bilinear collision operator localized around the local coordinate $\mathbf{r}[37,38,41]$. Combining (42) and (43) then yields

$$
\begin{aligned}
J\left(f_{1}, f_{1}\right)= & J_{0}\left(f_{1}^{(0)}, f_{1}^{(0)}\right)+\mu\left[J_{0}\left(f_{1}^{(0)}, f_{1}^{(1)}\right)\right. \\
& \left.+J_{0}\left(f_{1}^{(1)}, f_{1}^{(0)}\right)+J_{1}\left(f_{1}^{(0)}, \nabla_{\mathbf{r}_{1}} f_{1}^{(0)}\right)\right]+O\left(\mu^{2}\right),
\end{aligned}
$$

and the main difference with the classical expansion is the presence of the extra gradient terms $J_{1}\left(f_{1}^{(0)}, \nabla_{\mathbf{r}_{1}} f_{1}^{(0)}\right)$. 
At zeroth order, it is obtained that $J_{0}\left(f_{1}^{(0)}, f_{1}^{(0)}\right)=0$ and $f_{1}^{(0)}$ is taken to be the local Maxwellian

$$
f_{1}^{(0)}=n\left(\frac{m}{2 \pi k_{\mathrm{B}} T}\right)^{\frac{3}{2}} \exp \left(-\frac{m\left|\boldsymbol{c}_{1}-\boldsymbol{v}\right|^{2}}{2 k_{\mathrm{B}} T}\right),
$$

where $n, \boldsymbol{v}$, and $T$ are the local number density, fluid velocity, and temperature, following Enskog's method of expansion. In particular, the Maxwellian distribution is such that the local value of the complete internal energy density is recovered with $f_{1}^{(0)}$ thereby defining the local temperature $[38,41]$. The zeroth-order expansion $f_{2}^{(0)}$ of the pair distribution function $f_{2}$ is then in the form $[38,41,57]$

$$
f_{2}^{(0)}\left(\boldsymbol{x}_{1}, \boldsymbol{x}_{2}, t\right)=f_{1}^{(0)}\left(\mathbf{r}_{1}, \boldsymbol{c}_{1}, t\right) f_{1}^{(0)}\left(\mathbf{r}_{1}, \boldsymbol{c}_{2}, t\right) g_{2}\left(\mathbf{r}_{12}\right),
$$

where $g_{2}$ denotes the equilibrium correlation function

$$
g_{2}\left(r_{12}\right)=\exp \left[-\frac{\varphi_{12}\left(r_{12}\right)}{k_{\mathrm{B}} T\left(\mathbf{r}_{1}\right)}\right],
$$

keeping in mind that the macroscopic unknowns are locally uniform at zeroth order. At zeroth order, the Euler fluid equations are then recovered [36-42], using (45) and (46) and the macroscopic equations of Sec. III B.

At first order, in the Navier-Stokes regime, taking into account the operator decomposition (44), the integral equation for the perturbed distribution function $f_{1}^{(1)}$ is in the form

$$
\begin{gathered}
\partial_{t} f_{1}^{(0)}+\boldsymbol{c}_{1} \cdot \nabla_{\mathbf{r}_{1}} f_{1}^{(0)}-J_{1}\left(f_{1}^{(0)}, \nabla_{\mathbf{r}_{1}} f_{1}^{(0)}\right) \\
\quad=J_{0}\left(f_{1}^{(0)}, f_{1}^{(1)}\right)+J_{0}\left(f_{1}^{(1)}, f_{1}^{(0)}\right),
\end{gathered}
$$

and the time derivative is evaluated with Euler equations. This first-order equation is similar to the classical firstorder equations except that there are extra gradient terms $J_{1}\left(f_{1}^{(0)}, \nabla_{\mathbf{r}_{1}} f_{1}^{(0)}\right)$ arising from the first-order Taylor expansion of $f_{2}$ in the collision operator. The distribution $f_{1}^{(0)}$ is then uniquely determined by imposing Enskog-type constraints so that the true value of the local macroscopic variables $n, \boldsymbol{v}$, and $T$ (or equivalently $e$ ) are determined by $f_{1}^{(0)}$ alone [38,41]. The resulting macroscopic equations are of the Navier-StokesFourier type [36-42], with fluxes in the classical form (11) and (12). The resulting expressions of the transport coefficients in dense gases are found to be highly complex [36-42].

\section{E. Pressure and internal energy}

Using the zeroth-order expressions of the one- and twopoint distribution functions (45) and (46), the pressure is found in the form

$$
p=n k_{\mathrm{B}} T-\frac{n^{2}}{6} \int \varphi_{12}^{\prime}\left(r_{12}\right) r_{12} g_{2}\left(r_{12}\right) d \mathbf{r}_{12} .
$$

Integrating by part the integral in (49), we obtain that

$$
\begin{aligned}
& \int \varphi_{12}^{\prime}\left(r_{12}\right) r_{12} g_{2}\left(r_{12}\right) d \mathbf{r}_{12} \\
& =4 \pi \int \varphi_{12}^{\prime}\left(r_{12}\right) r_{12}^{3} g_{2}\left(r_{12}\right) d r_{12} \\
& =12 \pi k_{\mathrm{B}} T \int \mathfrak{f}_{12} r_{12}^{2} d r_{12}=3 k_{\mathrm{B}} T \int \mathfrak{f}_{12} d \mathbf{r}_{12},
\end{aligned}
$$

where $\mathfrak{f}_{12}$ is the Mayer function

$$
\mathfrak{f}_{12}=\exp \left(-\frac{\varphi_{12}}{k_{\mathrm{B}} T}\right)-1 .
$$

The state law may thus be written

$$
p=n k_{\mathrm{B}} T-\frac{n^{2}}{2} k_{\mathrm{B}} T \int \mathfrak{f}_{12} d \mathbf{r}_{12},
$$

and the internal energy is obtained with similar procedures

$$
e=\frac{3}{2} n k_{\mathrm{B}} T+\frac{1}{2} n^{2} \int \varphi_{12}\left(r_{12}\right) g_{2}\left(r_{12}\right) d \mathbf{r}_{12} .
$$

In order to recover the van der Waals equation of state, we may further assume that the interaction potential $\varphi_{12}$ is such that

$$
\varphi_{12}\left(r_{12}\right)= \begin{cases}+\infty & \text { if } 0 \leqslant r_{12} \leqslant \sigma, \\ \varphi_{12}\left(r_{12}\right)<\infty & \text { if } \sigma<r_{12},\end{cases}
$$

for some positive collision diameter $\sigma$. One may also assume that $\varphi_{12}$ is attractive $\varphi_{12}<0$ for $2 \sigma<r_{12}$ and increases towards zero as $r_{12} \rightarrow \infty$. We may then recover the van der Waals equation of state by decomposing the integral $\int \mathfrak{f}_{12} d r_{12}$ in two zones [58-60]. More specifically, one may write that

$$
\int \mathfrak{f}_{12} r_{12}^{2} d r_{12}=\int_{0}^{2 \sigma} \mathfrak{f}_{12} r_{12}^{2} d r_{12}+\int_{2 \sigma}^{\infty} \mathfrak{f}_{12} r_{12}^{2} d r_{12}
$$

use $\mathfrak{f}_{12}=-1$ over $(0,2 \sigma)$, and linearize the Mayer function $\mathfrak{f}_{12} \approx-\varphi_{12} / k_{\mathrm{B}} T$ for $2 \sigma<r_{12}$, to get that

$$
2 \pi k_{\mathrm{B}} T \int \mathfrak{f}_{12} r_{12}^{2} d r_{12} \approx-k_{\mathrm{B}} T \frac{16 \pi \sigma^{3}}{3}-2 \pi \int_{2 \sigma}^{\infty} \varphi_{12}\left(r_{12}\right) r_{12}^{2} d r_{12},
$$

and the corresponding pressure law reads

$$
p=n k_{\mathrm{B}} T+n^{2} k_{\mathrm{B}} T \frac{16 \pi \sigma^{3}}{3}+n^{2} 2 \pi \int_{2 \sigma}^{\infty} \varphi_{12}\left(r_{12}\right) r_{12}^{2} d r_{12} .
$$

Assuming then that the volume occupied by the particles is small, $n \frac{4 \pi \sigma^{3}}{3} \ll 1$, we obtain the state law

$$
p=\frac{n k_{\mathrm{B}} T}{1-\mathrm{b} n}-\mathrm{a} n^{2}
$$

with

$$
\mathrm{b}=4 \frac{4 \pi \sigma^{3}}{3}, \quad \mathrm{a}=-2 \pi \int_{2 \sigma}^{\infty} \varphi_{12}\left(r_{12}\right) r_{12}^{2} d r_{12},
$$

where $a$ is positive since the interaction potential is mainly negative as $\mathbf{r}$ increases. The van der Waals equation of state has thus been recovered using the traditional method of statistical physics [58-60].

\section{F. A second-order expansion}

The scaling of the generalized Boltzman equation (39) associated with the expansion (41) in the hydrodynamic regime may be written formally as

$$
\mu\left(\partial_{t} f_{1}+\boldsymbol{c}_{1} \cdot \nabla_{\mathbf{r}_{1}} f_{1}\right)=\int \theta_{12} f_{2} d \boldsymbol{x}_{2} .
$$

This shows that a second-order expansion of $f_{2}$, which leads to extra terms of order $O\left(\mu^{2}\right)$, should formally be taken into account in the macroscopic governing equations in a 
Navier-Stokes regime, keeping in mind that $f_{1}$ is developed with a first-order term $O(\mu)$ on the left-hand side. A secondorder expansion of the pair particle distribution $f_{2}$ is thus required and will yield the capillary terms in the macroscopic equations.

In order to obtain a second-order expansion of $f_{2}$, we first have to consider the one-point distribution function $f_{1}$. The distribution $f_{1}$ is expanded in the form

$$
f_{1}=f_{1}^{(0)}+\mu f_{1}^{(1)}+\mu^{2} f_{1}^{(2)}
$$

and this yields an expansion of $f_{1}\left(\mathbf{r}_{1}^{\prime}, \boldsymbol{c}_{1}\right)$ around $\boldsymbol{x}_{1}=\left(\mathbf{r}_{1}, \boldsymbol{c}_{1}\right)$ with $\delta \mathbf{r}_{1}=\mathbf{r}_{1}^{\prime}-\mathbf{r}_{1}$ in the form

$$
\begin{aligned}
f_{1}\left(\mathbf{r}_{1}^{\prime}, \boldsymbol{c}_{1}\right)= & f_{1}^{(0)}\left(\mathbf{r}_{1}, \boldsymbol{c}_{1}\right)+\mu\left[f_{1}^{(1)}\left(\mathbf{r}_{1}, \boldsymbol{c}_{1}\right)+\nabla f_{1}^{(0)}\left(\mathbf{r}_{1}, \boldsymbol{c}_{1}\right) \cdot \delta \mathbf{r}_{1}\right] \\
& +\mu^{2}\left[f_{1}^{(2)}\left(\mathbf{r}_{1}, \boldsymbol{c}_{1}\right)+\nabla f_{1}^{(1)}\left(\mathbf{r}_{1}, \boldsymbol{c}_{1}\right) \cdot \delta \mathbf{r}_{1}\right. \\
& \left.+\frac{1}{2} \nabla^{2} f_{1}^{(0)}\left(\mathbf{r}_{1}, \boldsymbol{c}_{1}\right): \delta \mathbf{r}_{1} \otimes \delta \mathbf{r}_{1}\right] .
\end{aligned}
$$

We now remark that both $f_{1}^{(2)}$ and $\nabla f_{1}^{(1)}$ are Burnett-type terms and should be discarded in a Navier-Stokes regime. Keeping only the $\nabla^{2} f_{1}^{(0)}$ second-order terms, we obtain a second-order expansion of $f_{1}$,

$$
\begin{aligned}
f_{1}\left(\mathbf{r}_{1}^{\prime}, \boldsymbol{c}_{1}\right)= & f_{1}^{(0)}\left(\mathbf{r}_{1}, \boldsymbol{c}_{1}\right)+\mu\left[f_{1}^{(1)}\left(\mathbf{r}_{1}, \boldsymbol{c}_{1}\right)+\nabla f_{1}^{(0)}\left(\mathbf{r}_{1}, \boldsymbol{c}_{1}\right) \cdot \delta \mathbf{r}_{1}\right] \\
& +\mu^{2} \frac{1}{2} \nabla^{2} f_{1}^{(0)}\left(\mathbf{r}_{1}, \boldsymbol{c}_{1}\right): \delta \mathbf{r}_{1} \otimes \delta \mathbf{r}_{1},
\end{aligned}
$$

that does not contain Burnett-type terms in the classical sense. The second-order expansion of $f_{2}$ is then obtained from that of $f_{1}(57)$ with the nonequilibrium expression

$$
f_{2}\left(\boldsymbol{x}_{1}, \boldsymbol{x}_{2}, t\right)=f_{1}^{(0)}\left(\boldsymbol{x}_{1}, t\right) f_{1}^{(0)}\left(\boldsymbol{x}_{2}, t\right) g_{2}\left(r_{12}\right),
$$

where $g_{2}\left(r_{12}\right)$ is the correlation function. In order to derive the modified macroscopic equations, the new expansion of $f_{2}$ must then be used with the general balance equations established in Sec. III B. Note that only the extra terms arising from the second-order terms of $f_{2}$ must be taken into account, and this is the object of the next section.

Although a nonequilibrium correlation function $g_{2}$ could in principle also be used, and could eventually depend on $T\left(\mathbf{r}_{2}\right)$, we will nevertheless use the correlation function (47) as if the temperature is locally uniform. There is indeed no clear expression for a nonequilibrium correlation function at interfaces, and many solutions have been proposed, e.g., using an average temperature, the center-of-mass temperature, or solving integral equations $[3,61,62]$. In addition, temperature variations during phase change are modest at variance with large density variations. Using a locally uniform correlation function $g_{2}$ will imply that the capillary coefficient is independent of temperature in agreement with the van der Waals and Rayleigh expressions obtained in the following and further corresponds to the simplified model presented in Sec. II.

\section{DERIVATION OF CAPILLARY FLUID EQUATIONS}

In this section, we derive the capillary fluid equations from the dense gas kinetic theory described in Sec. III. The method combines the dense fluid macroscopic equations obtained in Sec. III B as well as the second-order expansion of $f_{2}$. The second-order terms in $f_{2}$ are obtained from (58) and (57) and will yield the extra terms associated with capillarity. This method has intuitively been introduced by Rocard, who determined the force resulting from intermolecular potentials and deduced the Korteweg pressure tensor [34,35]. It is used systematically in this work for nonisothermal flows in order to derive the complete diffuse interface fluid equations.

\section{A. The Korteweg pressure tensor}

We consider the tensor $\mathcal{P}^{\mathrm{P}}$ arising from the interaction potential (32) and look for the extra terms with two spatial derivatives $\mathcal{P}^{\text {ex }}$ arising from the secondorder expansion of the distribution function $f_{2}\left(\boldsymbol{x}_{1}, \boldsymbol{x}_{2}, t\right)=$ $f_{1}^{(0)}\left(\boldsymbol{x}_{1}, t\right) f_{1}^{(0)}\left(\boldsymbol{x}_{2}, t\right) g_{2}\left(r_{12}\right)$. We use Taylor expansions and keep only the extra terms associated with two derivatives in the governing equations. The relevant integrand factor in (32) is expanded in the form

$$
\begin{aligned}
n_{2}\left[\mathbf{r}_{1}\right. & \left.-(1-\alpha) \mathbf{r}_{12}, \mathbf{r}_{1}+\alpha \mathbf{r}_{12}, t\right] \\
\approx & {\left[n\left(\mathbf{r}_{1}\right)-(1-\alpha) \nabla n\left(\mathbf{r}_{1}\right) \cdot \mathbf{r}_{12}\right.} \\
& \left.+\frac{1}{2}(1-\alpha)^{2} \nabla^{2} n\left(\mathbf{r}_{1}\right):\left(\mathbf{r}_{12} \otimes \mathbf{r}_{12}\right)\right] \\
& \times\left[n\left(\mathbf{r}_{1}\right)+\alpha \nabla n\left(\mathbf{r}_{1}\right) \cdot \mathbf{r}_{12}\right. \\
& \left.+\frac{1}{2} \alpha^{2} \nabla^{2} n\left(\mathbf{r}_{1}\right):\left(\mathbf{r}_{12} \otimes \mathbf{r}_{12}\right)\right] \times g_{2}\left(r_{12}\right) .
\end{aligned}
$$

Extracting the terms with two derivatives, the relevant integrands involves the factors

$$
\begin{aligned}
& \left.-\alpha(1-\alpha)\left[\nabla n\left(\mathbf{r}_{1}\right) \cdot \mathbf{r}_{12}\right)\right]^{2} \\
& \quad+\frac{1}{2}\left[(1-\alpha)^{2}+\alpha^{2}\right] n\left(\mathbf{r}_{1}\right) \nabla^{2} n\left(\mathbf{r}_{1}\right):\left(\mathbf{r}_{12} \otimes \mathbf{r}_{12}\right) .
\end{aligned}
$$

Incidentally, the $n^{2}$ term is already taken into account in the state law (49), and the odd terms yield zero contributions in $\mathcal{P}^{\text {ex }}$ but contribute in the dissipative fluxes [38,41].

Focusing on the first integrand, we note that

$$
\left.\left[\nabla n\left(\mathbf{r}_{1}\right) \cdot \mathbf{r}_{12}\right)\right]^{2}=\nabla n\left(\mathbf{r}_{1}\right) \otimes \nabla n\left(\mathbf{r}_{1}\right):\left(\mathbf{r}_{12} \otimes \mathbf{r}_{12}\right),
$$

and using $\int_{0}^{1} \alpha(1-\alpha) d \alpha=\frac{1}{6}$ we obtain a first extra contribution $\mathfrak{p}_{1}$ to the pressure tensor in the form

$$
\mathfrak{p}_{1}=\frac{1}{12} \int \frac{\varphi_{12}^{\prime}\left(r_{12}\right)}{r_{12}} \mathbf{r}_{12} \otimes \mathbf{r}_{12}\left[\nabla n \otimes \nabla n:\left(\mathbf{r}_{12} \otimes \mathbf{r}_{12}\right)\right] d \mathbf{r}_{12} .
$$

We may now use the identity (A3) from the Appendix in order to perform the integrations over $\mathbf{r}_{12} \in \mathbb{R}^{3}$, and we obtain that

$$
\mathfrak{p}_{1}=\frac{\varkappa}{6}\left(2 \nabla n \otimes \nabla n+|\nabla n|^{2} \boldsymbol{I}\right),
$$

where the capillarity coefficient is defined as

$$
\begin{aligned}
\varkappa & =\frac{1}{30} \int \varphi_{12}^{\prime}\left(r_{12}\right) g_{2}\left(r_{12}\right) r_{12}^{3} d \mathbf{r}_{12}, \\
& =\frac{2 \pi}{15} \int \varphi_{12}^{\prime}\left(r_{12}\right) g_{2}\left(r_{12}\right) r_{12}^{5} d r_{12} .
\end{aligned}
$$

Focusing next on the integrands, involving the Hessian matrix $\nabla^{2} n$ and using $\int_{0}^{1} \alpha^{2} d \alpha=\int_{0}^{1}(1-\alpha)^{2} d \alpha=\frac{1}{3}$, we obtain a second extra contribution $\mathfrak{p}_{2}$ to the pressure tensor in the form

$$
\mathfrak{p}_{2}=-\frac{n}{6} \int \frac{\varphi_{12}^{\prime}\left(r_{12}\right)}{r_{12}} \mathbf{r}_{12} \otimes \mathbf{r}_{12}\left[\nabla^{2} n:\left(\mathbf{r}_{12} \otimes \mathbf{r}_{12}\right)\right] d \mathbf{r}_{12} .
$$


Using again the identity (A3) in order to perform the integrations over $\mathbf{r}_{12} \in \mathbb{R}^{3}$, we obtain that

$$
\mathfrak{p}_{2}=-\frac{\varkappa}{3}\left(2 n \nabla^{2} n+n \Delta n \boldsymbol{I}\right) .
$$

Collecting previous results, the extra pressure tensor $\mathcal{P}^{\mathrm{ex}}$ is found in the form

$$
\mathcal{P}^{\mathrm{ex}}=\frac{\varkappa}{6}\left(2 \nabla n \otimes \nabla n+|\nabla n|^{2} \boldsymbol{I}-4 n \nabla^{2} n-2 n \Delta n \boldsymbol{I}\right) .
$$

We may then simplify $\mathcal{P}^{\text {ex }}$ with an equivalent expression $\overline{\mathcal{P}}^{\text {ex }}$; that is, we seek $\overline{\mathcal{P}}^{\text {ex }}$ such that

$$
\nabla \cdot \mathcal{P}^{\mathrm{ex}}=\nabla \cdot \overline{\mathcal{P}}^{\mathrm{ex}} .
$$

Expressing the divergence of the Hessian matrix contribution $n \nabla^{2} n$ with the help of differential identity (A5), we indeed obtain the simplified pressure tensor

$$
\overline{\mathcal{P}}^{\mathrm{ex}}=\varkappa\left(\nabla n \otimes \nabla n-\frac{1}{2}|\nabla n|^{2} \boldsymbol{I}-n \Delta n \boldsymbol{I}\right) .
$$

Introducing the mass based capillary coefficient $\varkappa_{m}=\varkappa / \mathrm{m}^{2}$ and using $\rho=m n$ we may rewrite the extra pressure tensor $\nabla \cdot \overline{\mathcal{P}}^{\text {ex }}$ as

$$
\overline{\mathcal{P}}^{\mathrm{ex}}=\varkappa_{m}\left(\nabla \rho \otimes \nabla \rho-\frac{1}{2}|\nabla \rho|^{2} \boldsymbol{I}-\rho \Delta \rho \boldsymbol{I}\right),
$$

which coincides with the Korteweg tensor $\overline{\mathcal{P}}^{\mathrm{ex}}=\mathcal{P}^{\mathrm{C}}$ given in (13).

\section{B. The capillarity coefficient}

The capillary coefficient has been found in the form (59), where $g_{2}\left(r_{12}\right)=\exp \left(-\varphi_{12} / k_{\mathrm{B}} T\right)$. We may integrate by part the integral in (59) to get that

$$
\varkappa=\frac{1}{6} k_{\mathrm{B}} T \int \mathfrak{f}_{12} r_{12}^{2} d \mathbf{r}_{12}=\frac{2 \pi}{3} k_{\mathrm{B}} T \int \mathfrak{f}_{12} r_{12}^{4} d r_{12},
$$

where $\mathfrak{f}_{12}$ is the Mayer function (50).

We may now simplify (62) in order to recover the van der Waals and Rayleigh capillarity coefficient. To this aim, we assume that the interaction potential is like (53). We simplify then the integral in (62) by neglecting the integral over $(0, \sigma)$ and by linearizing $\mathfrak{f}_{12}$ into $\mathfrak{f}_{12} \approx-\varphi_{12} / k_{\mathrm{B}} T$ for $r_{12}>\sigma$, and we obtain that

$$
\varkappa=-\frac{1}{6} \int_{r_{12}>\sigma} \varphi_{12} r_{12}^{2} d \mathbf{r}_{12},
$$

which coincides with the expression of van der Waals and Rayleigh [3]. Incidentally, considering, for instance, the attractive part of a Lennard-Jones potential $4 \varepsilon\left(\sigma / r_{12}\right)^{6}$, where $\varepsilon$ is the potential well depth, the integral over $(0, \sigma)$ is $k_{B} T \sigma^{5} / 5$ and that over $(\sigma,+\infty)$ is $4 \varepsilon \sigma^{5}$, and their ratio is $k_{\mathrm{B}} T / \varepsilon 20 \ll 1$ since $k_{\mathrm{B}} T / \varepsilon$ is typically of order unity.

We will use in the following the van der Waals and Rayleigh expression of the capillarity coefficient (63), which is independent of temperature and number density.

\section{The energy density}

We first investigate the extra terms $\mathcal{E}^{\text {ex }}$ arising from the energy density $\mathcal{E}^{\mathrm{P}}$ of potential origin (28) that involves an integral of the two-point distribution function $f_{2}$. Expanding the two-point distribution function $f_{2}=f_{1}\left(\boldsymbol{x}_{1}, t\right) f_{1}\left(\boldsymbol{x}_{2}, t\right) g_{2}\left(r_{12}\right)$ around $\mathbf{r}_{2}=\mathbf{r}_{1}+\mathbf{r}_{12}$, the relevant extra term involving two derivatives is in the form

$$
\mathcal{E}^{\mathrm{ex}}=\frac{1}{2} \int \varphi_{12}\left(r_{12}\right) n\left(\mathbf{r}_{1}\right) \frac{1}{2} \nabla^{2} n\left(\mathbf{r}_{1}\right):\left(\mathbf{r}_{12} \otimes \mathbf{r}_{12}\right) d \mathbf{r}_{12} .
$$

After some algebra, using the van der Waals and Rayleigh expression (63) for the capillarity coefficient, the resulting extra volumetric energy density $\mathcal{E}^{\text {ex }}$ is found to be

$$
\mathcal{E}^{\mathrm{ex}}=-\frac{1}{2} \varkappa n \Delta n \text {. }
$$

This type of energy density has been obtained in particular by Van der Waals [1] and Cahn and Hilliard [8]. In order to simplify (64) we may then use the identity

$$
\mathcal{E}^{\mathrm{ex}}=-\frac{1}{2} \varkappa n \Delta n=\frac{1}{2} \varkappa|\nabla n|^{2}-\frac{1}{2} \nabla \cdot(\varkappa n \nabla n),
$$

in order to recover an energy density in the form

$$
\overline{\mathcal{E}}^{\mathrm{ex}}=\frac{1}{2} \varkappa|\nabla n|^{2},
$$

which has also been obtained by Van der Waals [1] and Cahn and Hilliard [8], provided we take into account the residual $-\frac{1}{2} \nabla \cdot(\varkappa n \nabla n)$. By changing the energy density from $\mathcal{E}^{\text {ex }}=-\frac{1}{2} \varkappa n \Delta n$ into $\overline{\mathcal{E}}^{\mathrm{ex}}=\frac{1}{2} \varkappa|\nabla n|^{2}$ we have indeed to add an extra corrector term to the energy equation in the form

$$
\Xi=-\frac{1}{2}\left\{\partial_{t} \nabla \cdot(\varkappa n \nabla n)+\nabla \cdot[\nabla \cdot(\varkappa n \nabla n) v]\right\} .
$$

We may rewrite this corrector as the divergence of a corrector flux by using the mass conservation equation $\partial_{t} n=-\nabla(n \boldsymbol{v})$ and that the capillarity $\varkappa$ is constant. After some algebra, the corrector term $\Xi$ is rewritten as $\Xi=\nabla \cdot \mathfrak{q}_{0}$ where

$$
\mathfrak{q}_{0}=\frac{1}{2} \varkappa \nabla[n \nabla \cdot(n \boldsymbol{v})]-\frac{1}{2} \varkappa \nabla \cdot(n \nabla n) \boldsymbol{v} .
$$

Therefore, the extra flux $\mathfrak{q}_{0}$ must be taken into account, in addition to the extra terms involving two spatial derivatives that will arise from $\mathcal{Q}_{1}^{\mathrm{p}}$ and $\mathcal{Q}_{2}^{\mathrm{p}}$. For future use in the next section, we may further develop the extra flux $\mathfrak{q}_{0}$ in the form

$$
\begin{aligned}
\mathfrak{q}_{0}= & \varkappa n \nabla \cdot v \nabla n+\frac{1}{2} \varkappa n^{2} \nabla(\nabla \cdot v)+\frac{1}{2} \varkappa n(\nabla \boldsymbol{v})^{t} \cdot \nabla n \\
& +\frac{1}{2} \varkappa\left(\nabla n \otimes \nabla n+n \nabla^{2} n-|\nabla n|^{2} \boldsymbol{I}-n \Delta n \boldsymbol{I}\right) \boldsymbol{v} .
\end{aligned}
$$

The change of energy density from $\mathcal{E}^{\text {ex }}=-\frac{1}{2} \varkappa n \Delta n$ into $\overline{\mathcal{E}}^{\mathrm{ex}}=\frac{1}{2} \varkappa|\nabla n|^{2}$ has not been discussed in a satisfactory way in the literature. It has been advocated in previous work that $\mathcal{E}^{\text {ex }}$ may be transformed into $\overline{\mathcal{E}}^{\text {ex }}$ by integrating by part over "a large volume" with boundary conditions ensuring a null contribution of boundary terms. This is unsatisfactory since an energy density must be a local quantity, not defined over "a large volume" or else under the nonlocal influence of a far boundary with unclear definition and unclear boundary conditions. At variance, in this work, by properly taking into account the correction heat flux $\mathfrak{q}_{0}$, we clarify the local change of energy density from $\mathcal{E}^{\text {ex }}$ to $\overline{\mathcal{E}}^{\text {ex }}$.

\section{The Dunn and Serrin heat flux}

In order to evaluate the extra heat flux $\mathcal{Q}^{\text {ex }}$ arising from the second-order expansion of $f_{2}$ in $\mathcal{Q}_{1}^{\mathrm{p}}$ and $\mathcal{Q}_{2}^{\mathrm{p}}$ and using (58), we need to evaluate the gradients of $f_{1}^{(0)}$. After some algebra we note that

$$
\frac{\nabla f_{1}^{(0)}}{f_{1}^{(0)}}=\frac{m}{k_{\mathrm{B}} T}\left(\boldsymbol{c}_{1}-\boldsymbol{v}\right) \cdot \nabla \boldsymbol{v}+\frac{\nabla n}{n}-\left[\frac{3}{2}-\frac{m\left|\boldsymbol{c}_{1}-\boldsymbol{v}\right|^{2}}{2 k_{\mathrm{B}} T}\right] \frac{\nabla T}{T},
$$


where all gradients are of course evaluated at $\mathbf{r}_{1}$ and similarly that

$$
\begin{aligned}
\frac{\nabla^{2} f_{1}^{(0)}}{f_{1}^{(0)}}= & \frac{\nabla f_{1}^{(0)} \otimes \nabla f_{1}^{(0)}}{\left(f_{1}^{(0)}\right)^{2}}-\frac{m}{k_{\mathrm{B}} T} \boldsymbol{\nabla} \boldsymbol{v} \cdot \boldsymbol{\nabla v}+\frac{m}{k_{\mathrm{B}} T}\left(\boldsymbol{c}_{1}-\boldsymbol{v}\right) \cdot \nabla^{2} \boldsymbol{v}+\frac{\nabla^{2} n}{n}-\frac{\nabla n \otimes \nabla n}{n^{2}}-\frac{m}{k_{\mathrm{B}} T^{2}}\left[\left(\boldsymbol{c}_{1}-\boldsymbol{v}\right) \cdot \nabla \boldsymbol{v}\right] \otimes \nabla T \\
& -\frac{m}{k_{\mathrm{B}} T^{2}} \nabla T \otimes\left[\left(\boldsymbol{c}_{1}-\boldsymbol{v}\right) \cdot \nabla \boldsymbol{v}\right]-\left(\frac{3}{2}-\frac{m\left|\boldsymbol{c}_{1}-\boldsymbol{v}\right|^{2}}{2 k_{\mathrm{B}} T}\right) \frac{\boldsymbol{\nabla}^{2} T}{T}+\left(\frac{3}{2}-\frac{m\left|\boldsymbol{c}_{1}-\boldsymbol{v}\right|^{2}}{k_{\mathrm{B}} T}\right) \frac{\nabla T \otimes \nabla T}{T^{2}} .
\end{aligned}
$$

With $\mathcal{Q}_{1}^{\text {p }}$ given by (35), we need to select integrands obtained from a Taylor expansion of $f_{1}^{(0)}\left(\boldsymbol{x}_{1}\right) f_{1}^{(0)}\left(\boldsymbol{x}_{2}\right)$ that involve the peculiar velocity of the first particle $\boldsymbol{c}_{1}-\boldsymbol{v}$ as well as two spatial derivatives and that are even in $\mathbf{r}_{12}$. It appears, however, that there are not such terms in the Taylor expansion of $f_{1}^{(0)}\left(\mathbf{r}_{1}, \boldsymbol{c}_{1}, t\right) f_{1}^{(0)}\left(\mathbf{r}_{2}, \boldsymbol{c}_{2}, t\right)$ in such a way that there is no extra capillary heat flux arising from $\mathcal{Q}_{1}^{\mathrm{P}}$.

On the other hand, with $\mathcal{Q}_{2}^{\text {p }}$ given by (36), from the isotropy of space and velocity space, we need to select the integrands in the Taylor expansion of

$$
f_{1}^{(0)}\left[\mathbf{r}_{1}-(1-\alpha) \mathbf{r}_{12}, \boldsymbol{c}_{1}, t\right] f_{1}^{(0)}\left(\mathbf{r}_{1}+\alpha \mathbf{r}_{12}, \boldsymbol{c}_{2}, t\right),
$$

which involve two derivatives, are even with respect to $\mathbf{r}_{12}$, and are odd with respect to either $\boldsymbol{c}_{1}-\boldsymbol{v}$ or $\boldsymbol{c}_{2}-\boldsymbol{v}$. We first focus of integrands having such properties arising from the cross products of two first-order derivatives of $f_{1}^{(0)}$ at different points. These integrands are

$$
\begin{gathered}
-\alpha(1-\alpha) \frac{m}{k_{\mathrm{B}} T}\left(\boldsymbol{c}_{1}-\boldsymbol{v}\right) \cdot \nabla \boldsymbol{v} \cdot \mathbf{r}_{12} \frac{\nabla n \cdot \mathbf{r}_{12}}{n} \\
-\alpha(1-\alpha) \frac{m}{k_{\mathrm{B}} T}\left(\boldsymbol{c}_{2}-\boldsymbol{v}\right) \cdot \nabla \boldsymbol{v} \cdot \mathbf{r}_{12} \frac{\nabla n \cdot \mathbf{r}_{12}}{n},
\end{gathered}
$$

as well as other integrands still including the factor $\alpha(1-\alpha)$ and proportional to

$$
\begin{aligned}
& \frac{m}{k_{\mathrm{B}} T}\left(\boldsymbol{c}_{1}-\boldsymbol{v}\right) \cdot \nabla \boldsymbol{v} \cdot \mathbf{r}_{12}\left(\frac{3}{2}-\frac{m\left|\boldsymbol{c}_{2}-\boldsymbol{v}\right|^{2}}{2 k_{\mathrm{B}} T}\right) \frac{\nabla T \cdot \mathbf{r}_{12}}{T} \\
& +\frac{m}{k_{\mathrm{B}} T}\left(\boldsymbol{c}_{2}-\boldsymbol{v}\right) \cdot \nabla \boldsymbol{v} \cdot \mathbf{r}_{12}\left(\frac{3}{2}-\frac{m\left|\boldsymbol{c}_{1}-\boldsymbol{v}\right|^{2}}{2 k_{\mathrm{B}} T}\right) \frac{\nabla T \cdot \mathbf{r}_{12}}{T} .
\end{aligned}
$$

These later integrands yield vanishing contributions since

$$
\int\left(\frac{3}{2}-\frac{m\left|\boldsymbol{c}_{1}-\boldsymbol{v}\right|^{2}}{2 k_{\mathrm{B}} T}\right) f_{1}^{(0)} d \boldsymbol{c}_{1}=0
$$

Using then $\int_{0}^{1} \alpha(1-\alpha) d \alpha=\frac{1}{6}$ as well as the isotropy of the velocity space and elementary symmetries, we next obtain from the two former integrands a contribution $\mathfrak{q}_{1}$ for the heat flux in the form

$$
\begin{aligned}
\mathfrak{q}_{1}= & \frac{1}{12} \int \frac{\varphi_{12}^{\prime}\left(r_{12}\right)}{r_{12}} \mathbf{r}_{12} \mathbf{r}_{12} \cdot\left(\boldsymbol{c}_{1}-\boldsymbol{v}\right) \frac{m}{k_{\mathrm{B}} T}\left(\boldsymbol{c}_{1}-\boldsymbol{v}\right) \cdot \nabla \boldsymbol{v} \cdot \mathbf{r}_{12} \\
& \times \frac{\nabla n \cdot \mathbf{r}_{12}}{n} f_{1}^{(0)}\left(\mathbf{r}_{1}, \boldsymbol{c}_{1}\right) f_{1}^{(0)}\left(\mathbf{r}_{1}, \boldsymbol{c}_{2}\right) g_{2}\left(r_{12}\right) d \boldsymbol{c}_{1} d \mathbf{r}_{12} d \boldsymbol{c}_{2} .
\end{aligned}
$$

The integration over $\boldsymbol{c}_{2}$ is trivial and yields a factor $n$, the integration over $\boldsymbol{c}_{1}$ is performed using the reduced velocity $\left(c_{1}-v\right)\left(m / 2 k_{\mathrm{B}} T\right)^{1 / 2}$ and (A1) from the Appendix, and this yields

$\mathfrak{q}_{1}=\frac{n}{12} \int \frac{\varphi_{12}^{\prime}\left(r_{12}\right)}{r_{12}} \mathbf{r}_{12} \otimes \mathbf{r}_{12}\left[\nabla v:\left(\mathbf{r}_{12} \otimes \mathbf{r}_{12}\right)\right] g_{2}\left(\mathbf{r}_{12}\right) d \mathbf{r}_{12} \nabla n$.

Using the differential identity (A3) we then obtain that $\mathfrak{q}_{1}$ is in the form

$$
\mathfrak{q}_{1}=\frac{\varkappa}{6} n\left(\nabla \boldsymbol{v}+(\nabla \boldsymbol{v})^{t}+\nabla \cdot \boldsymbol{v I}\right) \nabla n,
$$

with the capillary coefficient given by (59).

We now focus on contributions arising from the secondorder derivatives $\nabla^{2} f_{1}^{(0)}$ in the Taylor expansion of $f_{1}^{(0)}\left[\mathbf{r}_{1}-\right.$ $\left.(1-\alpha) \mathbf{r}_{12}, \boldsymbol{c}_{1}, t\right] f_{1}^{(0)}\left(\mathbf{r}_{1}+\alpha \mathbf{r}_{12}, \boldsymbol{c}_{2}, t\right)$. The relevant integrands are those even with respect to $\mathbf{r}_{12}$ and odd with respect to either $c_{1}-\boldsymbol{v}$ or $c_{2}-\boldsymbol{v}$. These integrands are first

$$
\begin{aligned}
& \alpha^{2} \frac{2 m}{k_{\mathrm{B}} T}\left(\boldsymbol{c}_{1}-\boldsymbol{v}\right) \cdot \nabla \boldsymbol{v} \cdot \mathbf{r}_{12} \frac{\nabla n \cdot \mathbf{r}_{12}}{n} \\
& \quad+(1-\alpha)^{2} \frac{2 m}{k_{\mathrm{B}} T}\left(\boldsymbol{c}_{2}-\boldsymbol{v}\right) \cdot \nabla \boldsymbol{v} \cdot \mathbf{r}_{12} \frac{\nabla n \cdot \mathbf{r}_{12}}{n} \\
& \quad+\alpha^{2} \frac{m}{k_{\mathrm{B}} T}\left(\boldsymbol{c}_{1}-\boldsymbol{v}\right) \cdot \nabla^{2} \boldsymbol{v}:\left(\mathbf{r}_{12} \otimes \mathbf{r}_{12}\right) \\
& \quad+(1-\alpha)^{2} \frac{m}{k_{\mathrm{B}} T}\left(\boldsymbol{c}_{2}-\boldsymbol{v}\right) \cdot \nabla^{2} \boldsymbol{v}:\left(\mathbf{r}_{12} \otimes \mathbf{r}_{12}\right) .
\end{aligned}
$$

The two first integrands yield a contribution $\mathfrak{q}_{2}$ similar to $\mathfrak{q}_{1}$ but with the coefficient $-\frac{1}{3}$ instead on $\frac{1}{6}$,

$$
\mathfrak{q}_{2}=-\frac{\varkappa}{3} n\left(\nabla \boldsymbol{v}+(\nabla \boldsymbol{v})^{t}+\nabla \cdot \boldsymbol{v I}\right) \nabla n .
$$

Using the isotropy of space and elementary symmetries, the integrands associated with $\nabla^{2} v$ further yield the contribution

$$
\begin{aligned}
\mathfrak{q}_{3}= & -\frac{1}{12} \int \frac{\varphi_{12}^{\prime}\left(r_{12}\right)}{r_{12}} \mathbf{r}_{12} \mathbf{r}_{12} \cdot\left(\boldsymbol{c}_{1}-\boldsymbol{v}\right) \\
& \times \frac{m}{k_{\mathrm{B}} T}\left(\boldsymbol{c}_{1}-\boldsymbol{v}\right) \cdot \nabla^{2} \boldsymbol{v}:\left(\mathbf{r}_{12} \otimes \mathbf{r}_{12}\right) f_{1}^{(0)}\left(\mathbf{r}_{1}, \boldsymbol{c}_{1}\right) f_{1}^{(0)}\left(\mathbf{r}_{1}, \boldsymbol{c}_{2}\right) \\
& \times g_{2}\left(\mathbf{r}_{12}\right) d \boldsymbol{c}_{1} d \mathbf{r}_{12} d \boldsymbol{c}_{2} .
\end{aligned}
$$

The integration over $\boldsymbol{c}_{2}$ yield a factor $n$, the integration over $\boldsymbol{c}_{1}$ is obtained with (A1) from the Appendix so that

$$
\mathfrak{q}_{3}=-\frac{n^{2}}{12} \int \frac{\varphi_{12}^{\prime}\left(r_{12}\right)}{r_{12}} \mathbf{r}_{12} \mathbf{r}_{12} \cdot \nabla^{2} \boldsymbol{v}:\left(\mathbf{r}_{12} \otimes \mathbf{r}_{12}\right) g_{2}\left(\mathbf{r}_{12}\right) d \mathbf{r}_{12} .
$$

We may then use (A4) with $\nabla^{2} \boldsymbol{v}:\left(\mathbf{r}_{12} \otimes \mathbf{r}_{12} \otimes \mathbf{r}_{12}\right)=$ $\mathbf{r}_{12} \cdot \nabla^{2} v:\left(\mathbf{r}_{12} \otimes \mathbf{r}_{12}\right)$ to deduce that

$$
\mathfrak{q}_{3}=-n^{2} \frac{\varkappa}{6}[\Delta \boldsymbol{v}+2 \nabla(\nabla \cdot v)] .
$$


Finally, there are also integrands associated with temperature gradients in the combined form

$$
-\frac{2 m}{k_{\mathrm{B}} T}\left(\boldsymbol{c}_{1}-\boldsymbol{v}\right) \cdot \nabla \boldsymbol{v} \cdot \mathbf{r}_{12}\left(\frac{5}{2}-\frac{m\left|\boldsymbol{c}_{1}-\boldsymbol{v}\right|^{2}}{2 k_{\mathrm{B}} T}\right) \frac{\nabla T \cdot \mathbf{r}_{12}}{T}
$$

with a similar expression in terms of $\left(\boldsymbol{c}_{2}-\boldsymbol{v}\right)$, but these terms yield vanishing contributions since

$$
\int\left(\boldsymbol{c}_{1}-\boldsymbol{v}\right)\left(\frac{5}{2}-\frac{m\left|\boldsymbol{c}_{1}-\boldsymbol{v}\right|^{2}}{2 k_{\mathrm{B}} T}\right) f_{1}^{(0)} d \boldsymbol{c}_{1}=0 .
$$

and

$$
\int\left|\boldsymbol{c}_{1}-\boldsymbol{v}\right|^{2}\left(\frac{5}{2}-\frac{m\left|\boldsymbol{c}_{1}-\boldsymbol{v}\right|^{2}}{2 k_{\mathrm{B}} T}\right) f_{1}^{(0)} d \boldsymbol{c}_{1}=0 .
$$

Collecting previous results, we have obtained an extra total energy flux $\mathcal{Q}^{\text {ex }}+\mathcal{P}^{\text {ex }} \cdot v$ in the form

$$
\mathcal{Q}^{\mathrm{ex}}+\mathcal{P}^{\mathrm{ex}} \cdot \boldsymbol{v}=\mathfrak{q}_{0}+\mathfrak{q}_{1}+\mathfrak{q}_{2}+\mathfrak{q}_{3}+\mathcal{P}^{\mathrm{ex}} \cdot \boldsymbol{v},
$$

where $\mathfrak{q}_{0}$ arise from the change of energy density. The extra total energy flux $\mathcal{Q}^{\text {ex }}+\mathcal{P}^{\text {ex }} \cdot \boldsymbol{v}$ thus first reads

$$
\begin{aligned}
\mathcal{Q}^{\mathrm{ex}}+\mathcal{P}^{\mathrm{ex}} \cdot \boldsymbol{v}= & \varkappa n \nabla \cdot \boldsymbol{v} \nabla n+\frac{1}{2} \varkappa n^{2} \nabla(\nabla \cdot \boldsymbol{v})+\frac{1}{2} \varkappa n(\nabla \boldsymbol{v})^{t} \cdot \nabla n+\frac{1}{2} \varkappa\left(\nabla n \otimes \nabla n+n \nabla^{2} n-|\nabla n|^{2} \boldsymbol{I}-n \Delta n\right) \boldsymbol{v} \\
& -\frac{\varkappa}{6} n\left[\nabla \boldsymbol{v}+(\boldsymbol{\nabla} \boldsymbol{v})^{t}+\nabla \cdot \boldsymbol{v} \boldsymbol{I}\right] \nabla n-\frac{\varkappa}{6} n^{2}[\Delta \boldsymbol{v}+2 \boldsymbol{\nabla}(\boldsymbol{\nabla} \cdot \boldsymbol{v})]+\frac{\varkappa}{6}\left(2 \boldsymbol{\nabla} n \otimes \nabla n+|\nabla n|^{2} \boldsymbol{I}-4 n \nabla^{2} n-2 n \Delta n \boldsymbol{I}\right) \cdot \boldsymbol{v} .
\end{aligned}
$$

In order to simplify this expression we seek an equivalent expression $\overline{\mathcal{Q}}^{\text {ex }}$ of $\mathcal{Q}^{\text {ex }}$; that is, we seek a flux $\overline{\mathcal{Q}}^{\text {ex }}$ such that

$$
\boldsymbol{\nabla} \cdot\left(\mathcal{Q}^{\mathrm{ex}}+\mathcal{P}^{\mathrm{ex}} \cdot \boldsymbol{v}\right)=\nabla \cdot\left(\overline{\mathcal{Q}}^{\mathrm{ex}}+\overline{\mathcal{P}}^{\mathrm{ex}} \cdot \boldsymbol{v}\right) .
$$

This will indeed allow the simultaneous use of the simplified heat flux $\overline{\mathcal{Q}}^{\text {ex }}$ and simplified pressure tensor $\overline{\mathcal{P}}^{\text {ex }}$. In order to simplify the divergence of $\mathcal{Q}^{\text {ex }}+\mathcal{P}^{\text {ex }} \cdot \boldsymbol{v}$, the first term $\varkappa n \nabla \cdot v \nabla n$ is left unchanged since it corresponds to the Dunn and Serrin heat flux, all terms proportional to $v$ are regrouped, and the differential identity (A6) is used to transform the divergence of the term $-\frac{1}{6} \varkappa n \nabla \cdot v \nabla n$ in the second line that originates from $\mathfrak{q}_{1}+\mathfrak{q}_{2}$. After some lengthy algebra this yields the expression

$$
\begin{aligned}
\nabla \cdot\left(\mathcal{Q}^{\mathrm{ex}}+\mathcal{P}^{\mathrm{ex}} \cdot \boldsymbol{v}\right)= & \nabla \cdot\left[\varkappa n \nabla \cdot \boldsymbol{v} \nabla n-\frac{1}{3} \varkappa n \nabla \boldsymbol{v} \cdot \nabla n\right. \\
& +\frac{1}{3} \varkappa n(\nabla \boldsymbol{v})^{t} \cdot \nabla n-\frac{1}{6} n^{2} \Delta \boldsymbol{v} \\
& +\frac{1}{6} n^{2} \nabla(\boldsymbol{\nabla} \cdot \boldsymbol{v})+\varkappa(\nabla n \otimes \nabla n \\
& \left.\left.-\frac{1}{2}|\nabla n|^{2} \boldsymbol{I}-n \Delta n \boldsymbol{I}\right) \cdot \boldsymbol{v}\right] .
\end{aligned}
$$

Using then the differential identity (A7), the divergence of the second to fifth terms (four terms) in the right hand side miraculously vanishes, and we obtain

$$
\begin{aligned}
\nabla \cdot\left(\mathcal{Q}^{\mathrm{ex}}+\mathcal{P}^{\mathrm{ex}} \cdot \boldsymbol{v}\right) & =\nabla \cdot[\varkappa n \nabla \cdot \boldsymbol{v} \nabla n+\varkappa(\nabla n \otimes \nabla n \\
& \left.\left.-\frac{1}{2}|\nabla n|^{2} \boldsymbol{I}-n \Delta n \boldsymbol{I}\right) \cdot \boldsymbol{v}\right],
\end{aligned}
$$

so that the equivalent heat flux $\overline{\mathcal{Q}}^{\text {ex }}$ is given by

$$
\overline{\mathcal{Q}}^{\mathrm{ex}}=\varkappa n \nabla \cdot v \nabla n .
$$

Using the mass-based capillarity coefficient $\varkappa_{m}=\varkappa / m^{2}$, we thus obtain that the extra flux $\overline{\mathcal{Q}}^{\text {ex }}$ coincides with the Dunn and Serrin heat flux $\overline{\mathcal{Q}}^{\text {ex }}=\mathcal{Q}^{\mathrm{C}}$. The full capillary fluid equations (6)-(8) with the capillary fluxes (13) and (14) and the energy density (4) have finally been recovered. A key point in the simplification of the capillary heat flux was notably the use of the modified density energy $\overline{\mathcal{E}}^{\text {ex }}$ in order to compensate the complex corrections arising from $\mathcal{Q}_{2}^{\text {p }}$ by similar terms arising from $\mathfrak{q}_{0}$.

\section{DISCUSSION}

We have presented in the previous sections a molecular derivation of the complete diffuse interface fluid equations in the framework of the kinetic theory of dense gases. This derivation notably includes the van der Waals gradient energy, the Korteweg tensor (9), the Dunn and Serrin heat flux (10), the van der Waals capillarity coefficient, and the van der Waals equation of state. In this derivation, we have evaluated the transport fluxes $\mathcal{P}^{\text {ex }}$ and $\mathcal{Q}^{\text {ex }}$ from the kinetic theory and next their simplified versions $\overline{\mathcal{Q}}^{\text {ex }}$ and $\overline{\mathcal{P}}^{\text {ex }}$, although one could also alternatively investigate directly the force $\nabla \cdot \mathcal{P}^{\text {ex }}$ and the heat release $\nabla \cdot \mathcal{Q}^{\text {ex }}$. We now address the validity domain of the derivation from the kinetic theory, discuss various alternative models, and address some previous work.

The derivation of the van der Waals equation of state from statistical mechanics first requires us to obtain a pressure law in the form (51). It then uses an expansion of the second virial coefficient $\int \mathfrak{f}_{12} d \mathbf{r}_{12}$, involving the Mayer function and the full potential $\mathfrak{f}_{12}=\exp \left(-\frac{\varphi_{12}}{k_{B} T}\right)-1$, in the form $\frac{1}{2} k_{\mathrm{B}} T \int \mathfrak{f}_{12} d \mathbf{r}_{12}=k_{\mathrm{B}} T \mathrm{~b}-\mathrm{a}$. This expansion is closely associated with an estimate of the full potential as in (53). It next classically requires that the volume ratio $n \mathrm{~b}$ is small $n \mathrm{~b} \ll 1$ and makes use of the identity $(1-n \mathrm{~b})^{-1} \approx 1+n \mathrm{~b}$ [58-60]. An alternative derivation of the Van der Waals equation of state is discussed below. The domain of validity of the van der Waals equation of state, however, is much larger than that of the hypotheses made in the derivation and notably includes liquids where the volume ratio $n \mathrm{~b}$ is of order unity.

Similarly, the capillary fluid equations have been derived within the kinetic theory of moderately dense gases essentially assuming that $r_{0} \ll \lambda_{\mathrm{K}} \ll \Lambda$, where $r_{0}$ is the typical range of the interaction molecular potential, $\lambda_{\mathrm{K}}$ the mean-free path, and $\Lambda$ a hydrodynamic length. However, these capillary equation also apply to liquids where $r_{0} \approx \lambda_{\mathrm{K}}$. Therefore, as for the van der Waals equation of state, the domain of validity of the resulting equations is much larger than that of the kinetic theory of moderately dense gases. In the same vein, even though the second-order corrections have been included in a Navier-Stokes regime, the resulting equation may also be simplified by removing all dissipative terms, and we then recover the inviscid capillary fluid equations. The importance 
of these equations in conjunction with Hilbert's sixth problem has notably been emphasized by Gorban and Karlin [63,64] and Slemrod [65].

The derivation from the kinetic theory has been performed by simplifying the capillarity coefficient $\varkappa$ so that it is independent of $T$ and $n$. The temperature dependence of the capillary coefficients could still be investigated by using a nonequilibrium correlation function $g_{2}$. Since there is no clear expression for a nonequilibrium correlation function at interfaces, $g_{2}$ should be chosen carefully $[3,61,62]$. Preliminary analyses using the temperature at the center of mass lead to highly complex analytical calculations and new extra terms that do not reduce to the traditional thermodynamic models with variable capillarity coefficients. The dependence on $n$ may also be investigated but would require us to take into account the triplet distribution $f_{3}$ and to take into account triple collisions. Equations of states like the Redlich-Kwong equation of state $[66,67]$ could eventually be derived from a kinetic theory including triple collisions. Such equations of state, which are variants of that of van der Waals', and their corresponding thermodynamics [67] yield accurate results over the range of pressure, temperature, and mixture states of interest of many phenomena like supercritical combustion $[9,68]$.

A key argument in the derivation has been the use of a generalized Boltzmann equation with distribution functions that are evaluated at different spatial positions. In particular, analyses starting from the classical localized Boltzmann equation, however deep and interesting, can recover only Burnett-type terms but not the capillary terms. As notably pointed out by Gorban and Karlin [63], "terms which look similar to Korteweg's stress tensor have been recognized in the Chapman-Enskog many times" and "Everyone can compare the Korteweg stress with Burnett's equations." However, these Burnett-type terms traditionally involve pressure gradients or temperature gradients and are not capillary terms that require a nonlocal collision operator. In their prominent work, Gorban and Karlin have notably obtained the Burnett and super-Burnett as well as an infinitely accurate reduced hydrodynamic model starting from a linearized version of the 10-moment Grad fluid model [63]. Nevertheless, however deep and original these results are per se, they do not lead to the capillary fluid equations. In the same vein, Huang et al. [70] have obtained Burnett-type terms conducting a difficult mathematical analysis. Some of the resulting temperature gradients terms in their limiting macroscopic equations are still incorrectly interpreted as Korteweg-type terms. All these studies starting from the traditional Boltzmann equation are indeed missing the key tool of a nonlocal collision operator or a nonlocal force.

The Enskog-Vlasov and Boltzmann-Vlasov models, on the other hand, include a nonlocal force term and an Enskog-like or Boltzmann-like collision kernel. Such models have been used successfully to investigate phase change problems in a kinetic framework [21-32]. These models may be derived from the BBGKY hierarchy by decomposing the interaction full potential $\varphi_{12}$ in the form $\varphi_{12}=\varphi_{12}^{\text {att }}+\varphi_{12}^{\text {rep }}$ where $\varphi_{12}^{\text {att }}$ is a long-range weak attractive potential and $\varphi_{12}^{\text {rep }}$ a strongly repulsive hard core potential [21]. The weak potential $\varphi_{12}^{\text {att }}$ is usually assumed to have a range much greater than the interparticle distance and yields a Vlasov-type force when using the mean field approximation [21]. On the other hand, the repulsive potential operator term may be reduced into a collision term as in the derivation of Boltzmann operator from the hierarchy $[21,30,41,71]$. The correlation function in the Enskog collision term $g_{2}^{\text {rep }}$ is generally taken as the Heaviside function $g_{2}^{\text {rep }}=\mathrm{H}\left(r_{12}>\sigma\right)$ approximating $\exp \left(-\varphi_{12} / k_{\mathrm{B}} T\right) \approx$ $\exp \left(-\varphi_{12}^{\mathrm{rep}} / k_{\mathrm{B}} T\right)$, and the correlation function in the Vlasov term $g_{2}^{\text {att }}$ is generally arbitrary selected. Important issues are irreversibility and the $\mathrm{H}$ theorem as notably investigated by Beijeren and Ernst [72] and Grmela and García-Colín [73], as well as energy conservation as investigated by Benilov and Benilov [74]. In comparison with the hierarchy, information has been lost when transforming the collision term associated with the repulsive potential, when transforming the correlation function involving the full potential, and the second equation of the hierarchy governing $f_{2}$ is not available anymore for a kinetic derivation of the equation governing the potential internal energy $e^{\mathrm{p}}$. Nevertheless, Enskog-Vlasov and BoltzmannVlasov have many advantages like satisfying a $\mathrm{H}$ theorem, involving a nonlocal force, and being more convenient for analytical, numerical, or mathematical investigations while still keeping the main physical aspects of dense fluids [21-32].

Since the potential involved in the Vlasov term for such models is the attractive part $\varphi_{12}^{\text {att }}$, it is not possible anymore to proceed as in Sec. IIIE in order to derive the van der Waals equation of state. An elegant variant derivation is then possible by directly including a factor $(1-n \mathrm{~b})^{-1}$ in the correlation function $g_{2}^{\text {att }}$ of the Vlasov term as first suggested by Sobrino [21]. A more sophisticated variant due to Benilov and Benilov [74] uses a series expansion in terms of the number density, and it is also possible to modify $g_{2}^{\text {att }}$ in agreement with the Carnahan-Starling equation of state [69] as introduced by Frezzotti et al. [24]. This procedure directly yields a factor $(1-n b)^{-1}$ in the repulsive part of the state law. Incidentally, it would also yield such a factor in capillary terms obtained from the Vlasov force without further simplifications. In this work, we have used the exact equilibrium correlation function $g_{2}$ given by statistical mechanics (47) and followed the traditional derivation of the van der Waals equation of state from statistical mechanics using the full potential [58-60].

Capillary terms may also be obtained from the Vlasov force term, but only for their attractive part. This is essentially the result obtained by Rocard, who directly estimated the attractive force by expanding the distribution function and next derived the expression of the Korteweg tensor [34,35]. Piechór has also independently shown that the local Korteweg tensor may be obtained from the nonlocal Vlasov term [25]. Piechór has further investigated rigorously the mathematical properties of the Enskog-Vlasov kinetic equation and collision operators [25]. Piechór has then used a first-order expansion of the distribution function so that only the Navier-StokesFourier equations have been recovered [25]. The capillary terms indeed require the second-order expansion of $f_{2}$ introduced in Sec. IIIF. In a distinguished study, Takata and Noguchi have introduced a minimum BGK-Vlasov model in order to investigate phase transition in a van der Waals fluid [27]. Takata and Noguchi have investigated the limiting fluid for small Knudsen numbers in an isothermal framework. They have notably obtained a Laplacian-like capillary energy per unit mass, which yields a gradient square term after integra- 
tion by part, as well as a limiting Cahn-Hilliard-type model [27]. The same framework has next been used by Takata and coworkers in order to clarify the stability of uniform equilibrium states in the kinetic regime rather than that in the continuum limit [29].

Last, but not least, the remarkable numerical simulations by Barbante and Frezzotti [7] have shown a very good agreement between the solutions of the capillary fluid equations and that of a particular Enskog-Vlasov kinetic equation. This very good agreement, except in rarefied regions, is better understood knowing that the capillary fluid equations are a limiting model of kinetic equations and suggests new numerical simulations in order to deepen the analysis.

Concerning the numerical simulation of diffuse interface fluids, the evaluation of capillary coefficients is notably discussed by Lin et al. [75] and Stephan et al. [76]. These coefficients may also be directly evaluated from the kinetic expression or else be evaluated using molecular simulations [7]. Diffuse interfaces may notably be very thin at ordinary pressure, like a few nanometers, and it has been found convenient to numerically thicken such interfaces by increasing artificially the capillarity coefficient [6]. More sophisticated variants involve an increase of the capillarity coefficients in order to thicken interfaces while keeping constant the surface tension by further modifying the thermodynamics [6]. Energy conservation at the discrete level has also been found important in order to eliminate parasitic currents [77]. Another very interesting method is to increase the number of fluid variables by adding the gradient of density as a new unknown while discretizing capillary terms in an antisymmetric fashion $[12,78]$. Last, but not least, boundary conditions associated with diffuse interfaces may generally be derived from traditional variational procedures $[4,51,79]$. More sophisticated studies may take into account molecular interactions with a solid wall [80].

\section{CONCLUSION}

We have derived the full diffuse interface fluid equations from the kinetic theory of dense gases. Investigating more refined models would be of high scientific interest. This notably includes dense gas models including triple collisions so that the capillarity $\varkappa$ would depend on the number density $n$. It would also be of high scientific interest to investigate the situation where the capillarity coefficient $\varkappa$ depends on temperature $T$ using a properly chosen nonequilibrium correlation function $g_{2}$. The case of gas mixtures-for which there is also a failure of rational thermodynamics - could also be investigated using similar methods. Investigating fluctuations at both the kinetic and the capillary fluid levels would also be of high interest as well as boundary conditions with three phase lines. Numerical simulations would also be an outstanding tool for comparing nonlocal kinetic models and their corresponding macroscopic diffuse interface equations.

\section{ACKNOWLEDGEMENT}

This work was supported by the ANR INSIDE project, grant ANR-19-CE05-0037-02 of the French Agence Nationale de la Recherche.

\section{APPENDIX: TENSORIAL INTEGRAL AND DIFFERENTIAL IDENTITIES}

We summarize in this Appendix various tensorial integral relations used in the derivation of the capillary fluid equations. We denote by $z \in \mathbb{R}^{3}$ an integration variable that may represent either the rescaled particle velocity $(\boldsymbol{c}-\boldsymbol{v})\left(m / 2 k_{\mathrm{B}} T\right)^{1 / 2}$ or the relative position $\mathbf{r}_{12}=\mathbf{r}_{2}-\mathbf{r}_{1}$.

Letting $z=|z|$ for $z \in \mathbb{R}^{3}$, then for any isotropic function $\gamma(z)$ of $z$, we have

$$
\int z_{i}^{2} \gamma(z) d z=\frac{1}{3} \int z^{2} \gamma(z) d z, \quad 1 \leqslant i \leqslant 3,
$$

and this implies

$$
\int z(z \cdot \mathfrak{a}) \gamma(z) d z=\frac{1}{3} \int z^{2} \gamma(z) d z \mathfrak{a},
$$

for any vector $\mathfrak{a}$ independent of $z$. Similarly, we have [41]

$$
\int z \otimes z \gamma(z) d z=\frac{1}{3} \int z^{2} \gamma(z) d z \boldsymbol{I},
$$

where $\boldsymbol{I}$ is the identity tensor in $\mathbb{R}^{3}$ and $z \otimes z$ the tensor product of $z$ with itself having components $z_{i} z_{j}$ for $1 \leqslant i, j \leqslant 3$.

In the same vein, for any isotropic function $\gamma(z)$ of $z$ we have [41]

$$
\int z_{i}^{4} \gamma(z) d z=\frac{1}{5} \int z^{4} \gamma(z) d z, \quad 1 \leqslant i \leqslant 3,
$$

as well as

$$
\int z_{i}^{2} z_{j}^{2} \gamma(z) d z=\frac{1}{15} \int z^{4} \gamma(z) d z, \quad 1 \leqslant i<j \leqslant 3,
$$

As a consequence, for any second rank tensor $\mathbf{w}$ independent of $z$ we have

$$
\begin{aligned}
& \int \gamma(z) z \otimes z(z \otimes z: \mathbf{w}) d z \\
& =\frac{1}{15} \int z^{4} \gamma(z) d z\left(\mathbf{w}+\mathbf{w}^{t}+\mathbf{w}: I \mathbf{I}\right),
\end{aligned}
$$

where $\mathbf{w}: \mathbf{v}$ is the full contraction between the two tensors $\mathbf{w}$ and $\mathbf{v}$, so that $\mathbf{w}: I$ is the trace of the tensor $\mathbf{w}$, and $\mathbf{w}^{t}$ denotes the transpose of $\mathbf{w}$.

Finally, for any third-order gradient tensor like $\nabla^{2} v$ independent of $z$ and for any isotropic function $\gamma(z)$ we have

$$
\begin{aligned}
& \int \gamma(z) z\left(\nabla^{2} \boldsymbol{v}:(z \otimes z \otimes z)\right) d z \\
& =\frac{1}{15} \int z^{4} \gamma(z) d z[\Delta \boldsymbol{v}+2 \nabla(\nabla \cdot v)],
\end{aligned}
$$

where $\nabla^{2} v: z \otimes z \otimes z$ is the total contraction of $\nabla^{2} v$ with the tensor product $z \otimes z \otimes z$ given by $\nabla^{2} v: z \otimes z \otimes z=$ $\sum_{i j k} \partial_{\mathbf{r}_{j}} \partial_{\mathbf{r}_{k}} \boldsymbol{v}_{i} z_{i} z_{j} z_{k}$.

We now investigate various differential relations used in the derivation of the capillary fluid equations. The following relation is established for a scalar function $n(\mathbf{r})$ of $\mathbf{r} \in \mathbb{R}^{3}$ after 
integrations by parts:

$$
\boldsymbol{\nabla} \cdot\left(n \nabla^{2} n\right)=\boldsymbol{\nabla} \cdot\left(n \Delta n \boldsymbol{I}+|\boldsymbol{\nabla} n|^{2} \boldsymbol{I}-\nabla n \otimes \nabla n\right),
$$

where $\nabla n$ is the gradient of $n, \Delta n=\nabla \cdot \nabla n$ the Laplacian, and $\nabla^{2} n$ the Hessian matrix with components $\partial_{\mathbf{r}_{i}} \partial_{\mathbf{r}_{j}} n$ for $1 \leqslant$ $i, j \leqslant 3$.

In addition, for any scalar function $n(\mathbf{r})$ and any vector function $\boldsymbol{v}(\mathbf{r})$ of $\mathbf{r} \in \mathbb{R}^{3}$ one may establish after a few inte- gration by parts that

$$
\begin{aligned}
\nabla \cdot(n \nabla \cdot v \nabla n)= & \nabla \cdot\left[n \boldsymbol{\nabla} \boldsymbol{v} \cdot \nabla n+\left(|\nabla n|^{2} \boldsymbol{I}+n \Delta n \boldsymbol{I}\right.\right. \\
& \left.\left.-\nabla n \otimes \nabla n-n \nabla^{2} n\right) \cdot \boldsymbol{v}\right] .
\end{aligned}
$$

where $\nabla \boldsymbol{v}$ is the gradient matrix and $\boldsymbol{\nabla} \cdot \boldsymbol{v}$ the divergence of $\boldsymbol{v}$. Similarly, one may establish the following identity:

$$
\frac{1}{2} \nabla \cdot\left[n^{2} \nabla(\nabla \cdot v)\right]=\nabla \cdot\left(n \nabla \boldsymbol{v} \cdot \nabla n-n \nabla \boldsymbol{v}^{t} \cdot \nabla n+\frac{1}{2} n^{2} \Delta \boldsymbol{v}\right),
$$

where $\nabla \boldsymbol{v}^{t}$ denotes the transpose of the gradient matrix.
[1] J. D. van der Waals, Thermodynamische theorie der capillariteit in de onderstelling van continue dichtheidsverandering, Verhand. Kon. Akad. V Wetensch. Amst. Sect. 1 (1893) [in Dutch; English translation in J. Stat. Phys. 20, 200 (1979)].

[2] D. J. Korteweg, Sur la forme que prennent les équations du mouvement fluide si l'on tient compte de forces capillaires causées par les variations de densité considérables mais continues et sur la théorie de la capillarité dans l'hypothèse d'une variation continue de la densité, Arch. Neerl. Sci Exactes Nat. Série II 6, 1 (1901).

[3] J. S. Rowlinson and B. Widom, Molecular Theory of Capillarity (Dover Publications, Mineola, NY, 1989, 2002).

[4] P. Seppecher, Moving contact line in the Cahn-Hilliard theory, Int. J. Eng. Sci. 34, 977 (1996).

[5] D. M. Anderson, G. B. McFadden, and A. A. Wheeler, Diffuse interface methods in fluid mechanics, Ann. Rev. Fluid Mech. 30, 139 (1998).

[6] D. Jamet, Diffuse interface models in fluid mechanics, Semantic Scholar, Corpus Id. 18437499 (2005).

[7] P. Barbante and A. Frezzotti, A comparison of models for the evaporation of a Lennard-Jones fluid, Eur. J. Mech. B. Fluids 64, 69 (2017).

[8] J. W. Cahn, and J. E. Hilliard, Free energy of a nonuniform system. I. Interfacial free energy, J. Chem. Phys. 28, 258 (1958).

[9] P. Gaillard, V. Giovangigli and L. Matuszewski, A diffuse interface Lox/hydrogen transcritical flame model, Combust. Theory Model. 20, 486 (2016).

[10] J. E. Dunn and J. Serrin, On the thermomechanics of interstitial working, Arch. Rat. Mech. Anal. 88, 95 (1985).

[11] S. Gavrilyuk and S. Shugrin, Media with equations of state that depend on derivatives, J. Appl. Mech. Techn. Phys. 37, 179 (1996).

[12] S. Gavrilyuk and H. Gouin, Symmetric form of governing equations for capillary fluids, in Trends in Applications of Mathematics to Mechanics (Nice, 1998), Monogr. Surv. Pure Appl. Math., Vol. 106 (Chapman \& Hall/CRC, Boca Raton, FL, 2000), pp. 306-311.

[13] S. Benzoni-Gavage, R. Danchin, S. Descombes, and D. Jamet, Structure of Korteweg models and stability of diffuse interfaces, Interfaces Free Bound. 7, 371 (2005).

[14] D. Bresch, M. Gisclon, I. Lacroix-Violet, On Navier-StokesKorteweg and Euler- Korteweg systems: Application to quantum fluids models, Arch. Rat. Mech. Anal. 233, 975 (2019).
[15] S. Ono and S. Kondo, Molecular theory of surface tension in liquids, in Encyclopedia of Physics, edited by S. Flüdge (Springer-Verlag, Berlin, 1960), Vol. 10, pp. 134-280.

[16] R. Evans, The nature of the liquid-vapour interface and other topics in the statistical mechanics of non-uniform, classical fluids, Adv. Phys. 28, 143 (1979).

[17] A. Frezzotti and P. Barbante, Kinetic theory aspects of nonequilibrium liquid-vapor flows, Mech. Eng. Rev. 4, 16-00540 (2017).

[18] K. Aoki, Y. Sone, and T. Yamada, Numerical analysis of gas flows condensing on its plane condensed phase on the basis of kinetic theory, Phys. Fluids A 2, 1867 (1990).

[19] C. Cercignani, Rarefied Gas Dynamics (Cambridge University Press, Cambridge, 2000).

[20] A. Frezzotti, Boundary conditions at the vapor-liquid interface, Phys. Fluids 23, 030609 (2011).

[21] L. de Sobrino, On a kinetic equation of a van der Waals gas, Can. J. Phys. 45, 363 (1967).

[22] M. Grmela, Kinetic equation approach to phase transitions, J. Stat. Phys. 3, 347 (1971).

[23] J. Karkheck and G. Stell, Mean field kinetic theories, J. Chem. Phys. 75, 1475 (1981).

[24] A. Frezzotti, L. Gibelli, and S. Lorenzani, Mean field kinetic theory description of evaporation of a fluid into vacuum, Phys. Fluids 17, 012102 (2005).

[25] K. Piechór, Non-local Korteweg stresses from kinetic theory point of view, Arch. Mech. 60, 23 (2008).

[26] E. S. Benilov and M. S. Benilov, Semiphenomenological model for gas-liquid phase transition, Phys. Rev. E 93, 032148 (2016).

[27] S. Takata and T. Noguchi, A simple kinetic model for the phase transition of the van der Waals fluid, J. Stat. Phys. 172, 880 (2018).

[28] A. Frezzotti, L. Gibelli, D. A. Lockerby, and J. E. Sprittles, Mean field kinetic theory approach to evaporation of a binary liquid into vacuum, Phys. Rev. Fluids 3, 054001 (2018).

[29] S. Takata, T. Matsumoto, A. Hirahara, and M. Hattori, Kinetic theory for a simple modeling of a phase transition: Dynamics out of local equilibrium, Phys. Rev. E 98, 052123 (2018).

[30] A. C. Fowler, Phase transition in the Boltzmann-Vlasov equation, J. Stat. Phys. 174, 1011 (2019).

[31] E. S. Benilov and M. S. Benilov, The Enskog-Vlasov equation: 
A kinetic model describing gas, liquid, and solid, J. Stat. Mech. Theor. Exp. (2019) 103205.

[32] H. Struchtrup and A. Frezzotti, Grad's 13 moments approximation for Enskog-Vlasov equation, in 31st International Symposium on Rarefield Gas Dynamics RGD31, edited by Y. Zhang, D. R. Emerson, D. Lockerby, and L. Wu, AIP Conf. Proc. No. 2132 (AIP, New York, 2019), p. 120007.

[33] H. Hayakawa, S. Takada, and V. Garzó, Kinetic theory of shear thickening for a moderately dense gas-solid suspension: From discontinuous thickening to continuous thickening, Phys. Rev. E 96, 042903 (2017).

[34] Y. Rocard, Thermodynamique (Masson \& Compagnie, Paris, 1952).

[35] Y. Rocard, Thermodynamics, trans. C. R. S. Manders (Pitman, London, 1961).

[36] S. T. Choh, The kinetic theory of phenomena in dense gases, Ph.D. dissertation, University of Michigan, 1958.

[37] F. Andrews, On the solution of the BBGKY equations for a dense classical gas, J. Math. Phys. 6, 1496 (1965).

[38] L. S. García-Colín, M. S. Green, and F. Chaos, The ChapmanEnskog solution of the generalized Boltzmann equation, Physica 32, 450 (1966).

[39] M. H. Ernst, L. K. Haines, and J. R. Dorfmann, Theory of transport coefficients for moderately dense gases, Rev. Mod. Phys. 41, 296 (1969).

[40] S. Chapman and T. G. Cowling, The Mathematical Theory of Non-Uniform Gases (Cambridge University Press, Cambridge, 1970).

[41] J. H. Ferziger and H. G. Kaper, Mathematical Theory of Transport Processes in Gases (North-Holland, Amsterdam, 1972).

[42] J. R. Dorfman and H. van Beijeren, The kinetic theory of gases, in Statistical Mechanics, Part B, edited by B. J. Berne (Plenum Press, New York, 1977), pp. 65-179.

[43] P. G. de Gennes, Wetting: Statics and dynamics, Rev. Mod. Phys. 57, 827 (1985).

[44] W. Bu, D. Kim, and D. Vaknin, Density profiles of liquid/vapor interfaces away from their critical points, J. Phys. Chem. C 118, 12405 (2014).

[45] V. Giovangigli, Multicomponent Flow Modeling (Birkhaüser, Boston, 1999).

[46] E. Nagnibeda and E. Kustova, Non-Equilibrium Reacting Gas Flows (Springer Verlag, Berlin, 2009).

[47] M. Capitelli, D. Bruno, and A. Laricchiuta, Fundamental Aspects of Plasma Chemical Physics, Transport (Springer, New York, 2013).

[48] S. R. de Groot and P. Mazur, Non-Equilibrium Thermodynamics (Dover Publications, Mineola, NY, 1984).

[49] H. Freistühler and M. Kotschote, Phase-field and Kortewegtype models for the time-dependent flow of compressible twophase fluids, Archive Rat. Mech. Anal. 224, 1 (2017).

[50] M. Heida and J. Málek, On compressible Korteweg fluid-like materials, Int. J. Eng. Sci. 48, 1313 (2010).

[51] O. Souček, M. Heida, and J. Málek, On a thermodynamic framework for developing boundary conditions for Korteweg fluids, Int. J. Eng. Sci. 154, 103316 (2020).

[52] E. M. Lifshitz and L. P. Pitaevskii, Physical Kinetics, Landau and Lifshitz Course on Theoretical Physics, Vol. 10 (Elsevier, Oxford, 1981).

[53] N. N. Bogolioubov, Problems of a dynamic theory in statistical physics, in Studies in Statistical Mechanics, Vol. 1, edited by
J. de Boer and G. E. Ulhenbeck (North-Holland, Amsterdam, 1962).

[54] M. Born and H. S. Green, A General Kinetic Theory of Liquids (Cambridge University Press, Cambridge, 1946).

[55] J. G. Kirkwood, The statistical mechanical theory of transport processes, J. Chem. Phys. 14, 180 (1946).

[56] J. Yvon, La Théorie Statistique des Fluides et de l'Équation d'État, Actualités Scientifiques et Industrielles, Vol. 203 (Hermann, Paris, 1935).

[57] R. A. Piccirelli, Some properties of the long-time values of the probability densities for moderately dense gases, J. Math. Phys. 7, 922 (1966).

[58] R. Fowler and E. A. Guggenheim, Statistical Thermodynamics (Cambridge University Press, Cambridge, 1956).

[59] L. D. Landau and E. M. Lifshitz, Statistical Physics, Landau and Lifshitz Course on Theoretical Physics, Vol. 5 (Elsevier, Oxford, 1980).

[60] Diu, B. C. Guthmann, D. Lederer, and B. Roulet, Éléments de Physique Statistique (Hermann, Paris, 1989).

[61] J. Fisher and M. Methfessel, Born-Green-Yvon approach to the local densities of a dilute fluid at interfaces, Phys. Rev. A 22, 2836 (1980).

[62] T. R. Osborn and C. A. Croxton, Monotonic and oscillatory profiles at the free liquid surface for simple atomic fluids, Mol. Phys. 40, 1489 (1980).

[63] A. N. Gorban and I. V. Karlin, Beyond Navier-Stokes equations: Capillary of ideal fluids, Contemp. Phys. 58, 70 (2017).

[64] A. N. Gorban and I. V. Karlin, Hilbert's sixth problem: Exact and approximate hydrodynamic manifolds for kinetic equations, Bull. Amer. Math. Soc. 51, 187 (2014).

[65] M. Slemrod, Hilbert's sixth problem and the failure of the Boltzmann to Euler limit, Phil. Trans. R. Soc. A 376, 20170222 (2018).

[66] O. Redlich and J. N. S. Kwong, On the thermodynamics of solutions. V, An equation of state. Fugacities of gaseous solutions, Chem. Rev. 44, 233 (1949).

[67] V. Giovangigli and L. Matuszewski, Supercritical fluid thermodynamics from equations of state, Physica D 241, 649 (2012).

[68] V. Giovangigli, L. Matuszewski, and F. Dupoirieux, Detailed modeling of transcritical planar $\mathrm{H}_{2}-\mathrm{O}_{2}-\mathrm{N}_{2}$ flames, Comb. Theory Mod. 15, 141 (2011).

[69] N. Carnahan and K. E. Starling, Equation of state for nonattracting rigid spheres, J. Chem. Phys. 51, 635 (1969).

[70] F. Huang, Y. Wang, Y. Wang, and T. Yang, Justification of limit for the Boltzmann equation related to Korteweg theory, Q. Appl. Math. 74, 719 (2016).

[71] H. Grad, Principles of the kinetic theory of gases, in Handbuch der Physik, Vol. 12 (Springer, Berlin, 1958), pp. 205-294.

[72] H. Van Beijeren and M. H. Ernst, The modified Enskog equation, Physica A 68, 437 (1973).

[73] M. Grmela and L. S. García-Colín, Compatibility of the Enskog kinetic theory with thermodynamics I, Phys. Rev. A 22, 1295 (1980).

[74] E. S. Benilov and M. S. Benilov, Energy conservation and $\mathrm{H}$ theorem for the Enskog-Vlasov equation, Phys. Rev. E 97, 062115 (2018).

[75] H. Lin, Y.-Y. Duan, and Q. Min, Gradient theory modeling of surface tension for pure fluids and binary mixtures, Fluid Phase Equil. 254, 75 (2007). 
[76] S. Stephan, K. Labgenbach, and H. Hesse, Interfacial properties of binary Lennard-Jones mixtures by molecular simulation and density gradient theory, J. Chem. Phys. 150, 174704 (2019).

[77] D. Jamet, O. Lebaigue, N. Coutris, and J. M. Delhaye, The second gradient method for the direct numerical simulation of liquid-vapor flows with phase change, J. Comput. Phys. 169, 624 (2001).

[78] D. Bresch, F. Couderc, P. Noble, and J.-P. Vila, A generalization of the quantum Bohm identity: Hyperbolic CFL condition for Euler-Korteweg equations, C. R. Acad. Sci. Paris Série I 354, 39 (2008).

[79] P. Seppecher, Étude des conditions aux limites en théorie du second gradient: Cas de la capillarité, C. R. Acad. Sci. Paris Série II 309, 497 (1989).

[80] H. Gouin and W. Kosiński, Boundary conditions for a capillary fluid in contact with a wall, Arch. Mech. 50, 907 (1998). 